\title{
Étude sur les pâturages et les questions fourragères en République Centrafricaine
}

\author{
par J. KOECHLIN
}

L'élevage des bovins en République Centrafricaine est pratiqué selon deux modalités :

10 Des groupes nomades et semi-nomades Borroros et quelques sédentaires Foulbés possèdent d'importants troupeaux répartis dans trois secteurs :

a) Une zone située le long de la frontière camerounaise et centrée autour des postes de Bouar, Baboua, Bocaranga (250.000 têtes).

b) A l'est de Bambari, une zone située entre les cours inférieurs de la Ouaka et de la Kotto (100.000 têtes).

c) A l'est de Carnot, une zone a été réservée à l'élevage entre la Lobaye et le village de Zaorosoungou (12.000 têtes).

Tous ces éleveurs possèdent exclusivement du bétail de race Zébu, Borroro ou Foulbé, donc très sensible à la trypanosomiase. Cette maladie limite actuellement l'extension des zones d'élevage et donne un intérêt accru aux problèmes de pâturages.

La lutte sanitaire est activement menée par le Service de l'Elcvage qui procède également à des assainissements par destruction des galeries forestières.

$2^{\circ}$ Le Service de l'Elevage met en place chez les sédentaires agriculteurs de petits noyaux de bétail trypanotolérant (races Baoulé ou des Lagunes). II existe environ actuellement 2.500 têtes réparties sur l'ensemble du territoire.

3o Signalons enfin que l'installation de ranchs d'élevage est actuellement envisagée.

Les incidences économiques de l'élevage pour la République Centrafricaine sont considérables:

Texte publié par le Ministère de la Coopération en auôl 1961.

Rev. Elev. Méd. Vét. Pays trop., 1962, 15, nº 1.
- L'élevage sédentaire est susceptible de fournir un appoint alimentaire important à des populations dont la ration est généralement carencée en protéines et dont les revenus sont limités.

- La présence des éleveurs nomades provoque la création d'échanges commerciaux non négligeables avec les populations sédentaires, mais surtout, le troupeau représente un capital considérable, de l'ordre de 3 milliards de francs CFA, encore insuffisamment exploité. Le marché local de la viande se développe rapidement, et il n'est pas satisfait par la production centrafricaine. Les possibilités d'exportation vers certains pays voisins sont importantes et la R. C. A. participe peu à ce courant commercial.

Environ 10.000 têtes de bétail sont commercialisées chaque année dans le secteur de Bovar, et entre 3 et 5.000 sur celui de Bambari. Du fait des abattages non contrôlés, ces chiffres peuvent être notablement augmentés.

Mais Bangui consomme environ 2.500 tonnes de viande annuellement et l'on importe du Tchad chaque année à peu près 25.000 têtes de bétail de boucherie.

La production centrafricaine doit donc être développée et les investissements à consentir dans ce domaine se trouveront parfaitement justifiés.

Il devient en particulier urgent de se préoccuper des pâturages jusqu'ici laissé au second plan devant l'urgence des questions sanitaires.

Une bonne connaissance de la végétation ef de sa valeur est, en effet, une base indispensable à toute tentative d'extension des zones d'élevage. En outre, dans les régions actuellement exploitées, des phénomènes de dégradation se manifestent qui réduisent considérablement la productivité des pâturages. 
1 Climat guinéen.

2 Climat soudano-guinéen, à tendance préforestière.

3 Climat soudano-guinéen typique.

4 Climat soudano-sahélien.
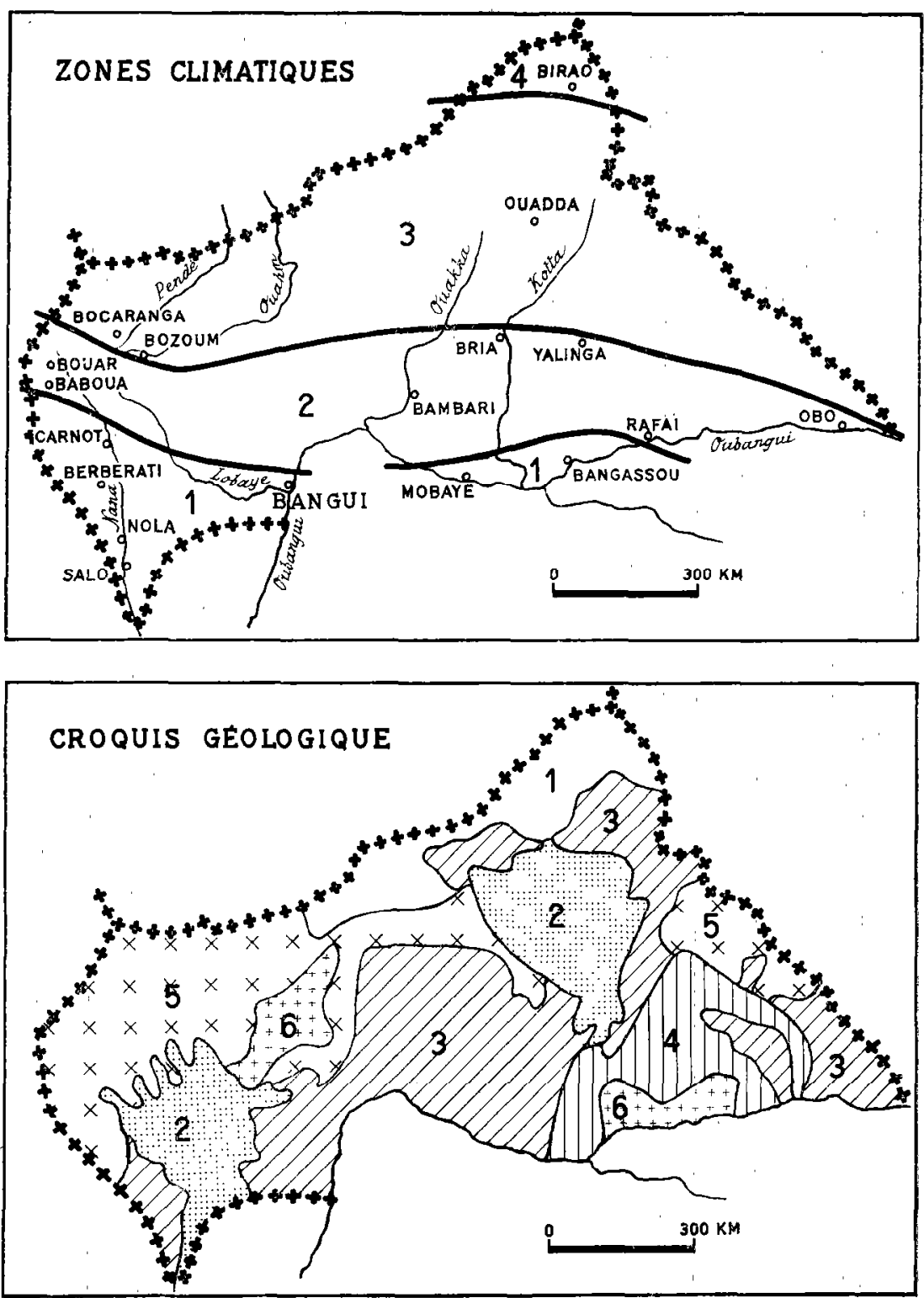

1 Alluvions quaternaires de la cuvette.

2 Grès du Continental terminal. Sols sableux.

3 Séries quartzo-schisteuses, avec principalement des quarts ou des grès quartziteux. Sols argileux à sabloargileux.

4 Séries schislo-gréseuses; sols comme ci-dessus.
5 Granito-gneiss. Sols variables avec souvent des horizons. superficiels grossiers ou d'abondants éléments latéritiques.

6 Roches métamorphiques (complexes à Pyroxènes, Amphibolites ef Pyroxénites). Sols argileux et latéritisés. Ce croquis, très sommaire, ne donne que la localisation des principales zones d'affleurement. 


\section{LES DIFFÉRENTS TYPES DE PATURAGES EN RÉPUBLIQUE CENTRAFRICAINE}

Il n'est pas question ici d'en faire une étude détaillée mais d'indiquer pour chacun les principales espèces constituantes de la flore et leur valeur fourragère.

La végétation dépend en premier lieu du climat, qui en détermine la forme (forêt, savane...) et de la nature du sol, responsable de la différenciation des groupements végétaux.

Les régions de savane de la R. C. A. sont situées principalement sous un climat de type soudano-guinéen : on peut distinguer une zone située au sud d'une ligne approximative Bozoum, Bria, Yalinga, Obo, recevant de 1.350 à $1.600 \mathrm{~mm}$ de pluie avec environ 4 mois de saison sèche, de mi-novembre à mi-mars. C'est un climat encore nettement préforestier. Au nord de cette ligne, par contre, c'est typiquement le domaine de la savane boisée, avec 1.200 à $1.400 \mathrm{~mm}$ de pluie, parfois $900 \mathrm{~mm}$ seulement vers le nord, et une saison sèche de 5 mois à 5 mois et demi, de début novembre à mi-avril environ.

Le climat guinéen forestier remonte approximativement jusqu'à la hauteur de Carnot et pénètre également sur le territoire de la $R$. C. A. entre Mobaye et Rafaï. II tombe environ $1.500 \mathrm{~mm}$ de pluie dans la région de Carnot-Nola, 2.000 dans celle de Bangassou. La saison sèche dure de 1 à 3 mois, mais les petifes pluies y restent cependant assez fréquentes.

Signalons enfin le climat soudano-sahélien qui règne dans l'extrême nord du Territoire (Birao) avec moins de $900 \mathrm{~mm}$ de pluie et 6 mois de saison sèche.

Les sols sont en relation directe avec la géologie. Suivant une classification établie par le Service de l'Elevage (Dr. J. Laurent) avec l'aide de $P$. Benoît-Janin et adaptée aux problèmes pastoraux, on peut distinguer les types suivants:

10 Sols sableux sur grès, pauvres en éléments minéraux ( $\mathrm{C} a$ et $\mathrm{P}$ en particulier) perméables, lessivés. Occupent des superficies importantes en Haute-Sangha (Grès de Carnot) ef autour de Ouadda, dans le N.-E.

2a, Sols sableux à sablo-argileux, sur quartzites, encore perméables, et pauvres en calcium. Se rencontrent principalement au sud de Berbérati et autour de Bambari.
30 Sols plus argileux, avec horizon superficiel grossier, formés à partir de granito-gneiss, teneurs minérales variables, érosion à craindre, régions de Bouar et Bocaranga surtout.

40 Sols de texture variée, mais généralement argileux, sur roches métamorphiques; assez bonne teneur en calcium, érosion peu à craindre, mais présence fréquente de gravillons latéritiques.

5o Sols très argileux ef latérisés, sur roches métamorphiques riches en éléments noirs, teneurs calciques élevées.

Ces deux derniers types de sols se rencontrent dispersés un peu partout sous forme de taches plus ou moins importantes.

\section{A) Appréciation de la valeur d'un pâturage}

Dans les savanes soudano-guinéennes ou guinéennes, les graminées sont, pratiquement les seules piantes appétées par. les bovins. II s'agit en grande majorité d'espèces pérennes. Avec la croissance (elles atteignent généralement un à plusieurs mètres de haut), elles se lignifient, se chargent de silice et deviennent rapidement inconsommables.

Ceci implique obligatoirement une exploitation du pâturage en rotation, ou, du moins, d'une façon assez continue pour que la repousse d'herbe jeune se renouvelle constamment. Pour la saison sèche, on est obligé de recourir au feu qui détruit les vieilles herbes et provoque un regain de pousses vertes.

Pour un type de pâturage, la repousse de l'herbe est fonction de nombreux facteurs : nature de la plante et de son enracinement sol, richesse chimique et capacité en eau - climat, quantité et répartition des pluies - fréquence du pâturage, c'est-à-dire de la coupe.

L'appréciation du rendement d'un pâturage devra donc être basée sur le rendement de coupes périodiques, effectuées avec la même fréquence que celle des rotations du troupeau. Une coupe globale effectuée à la fin de la période de croissance aurait peu de signification (1).

L'appétabilité des espèces et leur valeur bromatologique sont indiquées par l'étude du compor-

(1) Voir $\S I V$, Etude des pâturages sur les statiorıs d'élevage. 
tement des animaux et des résultats de l'analyse fourragère. II convient cependant de définir exactement la valeur des renseignements que l'on peut attendre de cette dernière.

l_e problème de l'échantillonnage est primordial : la composition chimique d'une plante est en effet, variable en fonction de son âge, de son stade de développement ef du niveau auquel est fait le prélèvement : extrémité ou base des feuilles ou des tiges par exemple. II faut de plus situer exactement le milieu dans lequel est fait le prélèvement : nature du sol et type de la végétation défini par l'analyse floristique précise du groupement végétal. Le prélèvement à analyser doit enfin être réalisé d'une façon telle qu'il représente un échantillonnage valable du type de pâturage étudié.

Ce n'est que dans ces conditions que des analyses auront une signification réelle, qu'elles pourront être comparées entre elles et que ces résultats seront applicables à des zones ou à des types de pâturage qu'il sera possible de définir exactement. Des prélèvements isolés, tels que ceux dont il sera fait état plus loin, ne sont susceptibles d'apporter que des indications d'un ordre très général.

L'étude de la structure anatomique des feuilles de graminées est également susceptible de donner des indications sur leur digestibilité, leur valeur alimentaire globale et leur mode de végétation. La digestibilité et la valeur alimentaire sont, en effet, proportionnelles à la teneur en éléments parenchymateux et inversement à celle des éléments sclérifiés. De même, la capacité de croissance sera d'autant plus grande que le tissu assimilateur chlorophyllien sera plus développé (2).

On peuł ainsi établir une classification des graminées en fonction de ces critères. Elle est d'ailleurs confirmée par les tests d'appétabilité : on trouve, en effet; en tête des plantes comme Brachiaria brizantha ou Beckeropsis uniseta (type A, fig. 1) puis la plupart des Andropogon ef des Hyparrhenia (type B) et enfin des plantes fourragères médiocres telles que les Loudetia ou les Sporobolus (type C).

(2) Méthode étudiée par KIWARK et DUVIGNEAUD au Bas-Congo (ex-Belge).

\section{B) Les principaux types de végétation}

\section{1o La végéfation naturelle.}

Nous nous attacherons à définir plutôt des types de pâturages que des types botaniques de végétation, parfois complexes à analyser pour des non-spécialistes.

En pratique, la reconnaissance de certaines espèces typiques de conditions écologiques. définies, suffit pour caractériser un pâfurage.

\section{A. - Savanes arbustives ou boisées sur terrains secs.}

En ce qui concerne l'élevage, la strate ligneuse n'intervient que par sa densité, qui peut créer un obstacle mécanique, et l'abondance de certaines essences comme Annona arenaria, Burkea africana, Hymenocardia acida, susceptibles de rejeter fortement et de provoquer un embuissonnement des parcours.

a) Savanes sur sols argileux à argilo-sableux.

Tapis herbacé généralement dense ef élevé constitué en majeure partie d'Andropogonnées. appartenant aux genres Hyparrhenia et Andropogon avec, en proportions variables, des espèces comme Setaria splendida, Beckeropsis uniseta, ou des Loudetia. Ceux-ci mis à part, il s'agit toujours d'espèces bien appétées, réagissant bien au pâturage et donnant des repousses correctes en saison sèche. Les carences minérales sont peu à craindre dans de tels pâturages.

Diverses espèccs pcuvent assurer la dominance:

- Hyparrhenia diplandra : une des espèces les plus répandues, sur sols assez argileux et profonds, avec une bonne économie en eau, ou bien pourvus en matière organique. De telles savanes se rencontrent par exemple dans les. régions de Bouar, Bambari (Langandi) ou Bossembélé. Ce sont d'excellents pâturages donnant une bonne repousse en saison sèche.

- Andropogon gayanus, A. gabonensis, Beckeropsis uniseta. Ce sont de très grandes herbcs généralement associées sur les sols frais et riches en matières organiques (alluvions). Elles occupent rarement des grandes superficies. Très appétées, elles repoussent bien en saison sèche, mais résistent mal à un pâturagc intensif.

- Andropogon tectorum a des caractéristiques semblables, mais se rencontre dans les savanes à couvert arboré dense, associée à des plantes 


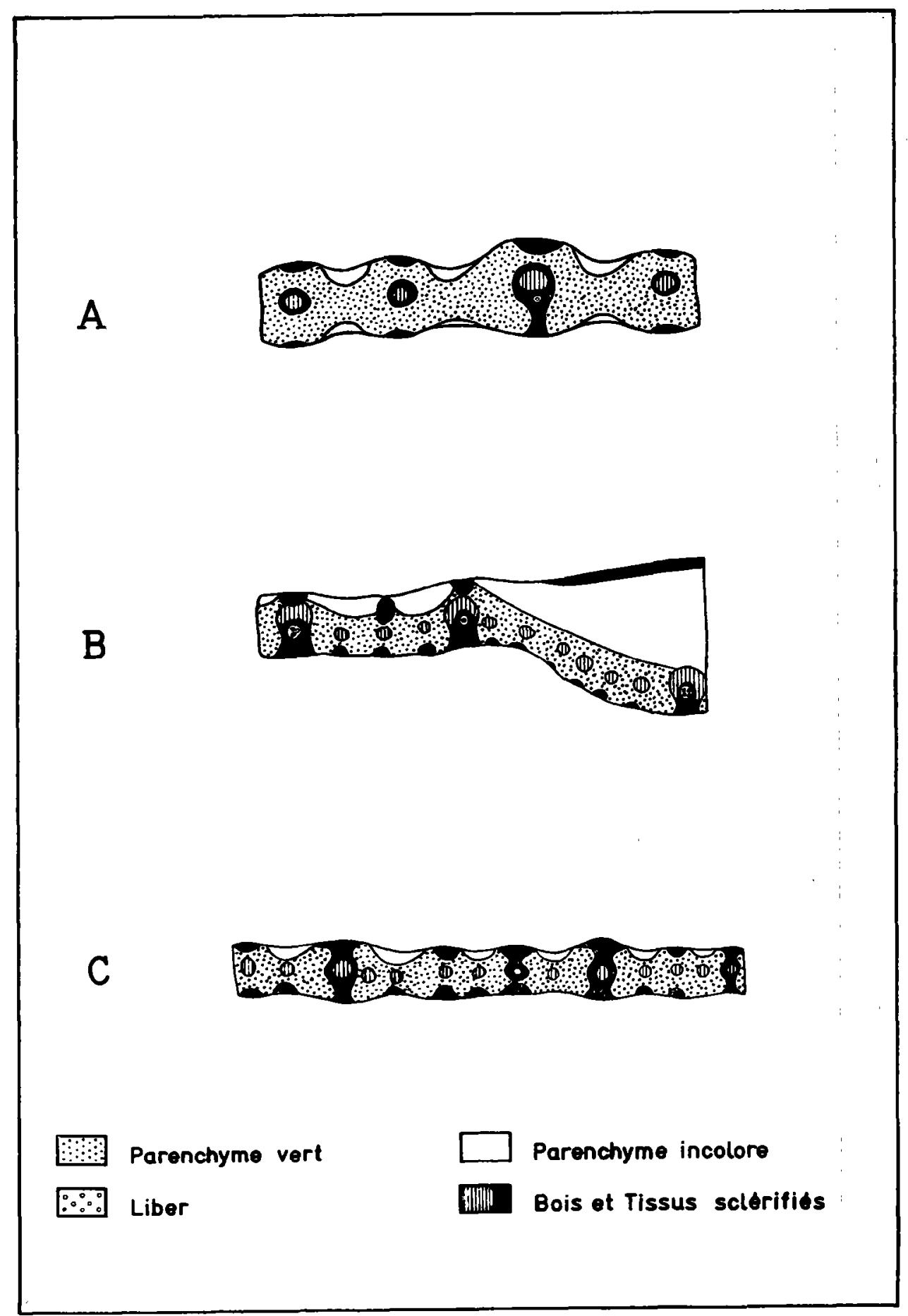

Fig. 1. - Types de structures anctomiques des graminées. 
de faible valeur fourragère comme Nephrolepis cordifolia (fougère) ou Afromomum sp.

- Elymandra androphila est souvent abondante sur les sols assez sableux de la région de Carnot, ou autour de Bossembélé. C'est une remarquable graminée fourragère réagissant très bien à l'action du bétail ; son comportement en saison sèche n'est pas encore connu.

- Andropogon schirensis, de taille plus réduite, est l'indice de sols 'secs ou peu profonds sur lesquels la repousse de saison sèche sera médiocre. On peut rencontrer cette espèce en peuplements plus ou moins étendus, sur des sommets de colline, sur sols caillouteux, ou lorsqu'il existe une cuirasse ferrugineuse à faible profondeur.

- Hyparrhenia gracilenscens ef $H$. rufa se rencontrent fréquemment dans les sols alluviaux. La première occupe plutôt des terrains légers ; c'est une plante annuelle qui fournira un bon pâturage de saison des pluics. La dcuxic̀me cst plus exigeante et occupe généralement des sols plus lourds; elle fournit un excellent pâturage.

Ces différentes espèces peuvent naturellement cohabiter dans la même station : d'après leurs proportions, il sera possible de déterminer les caractéristiques du pâturage.

b) Savanes sur sols sableux à sablo-argileux paurres.

Tapis herbacé de hauteur et de densité variable, riche en Arundinellées (Loudetia, Tristachya). Ce sont des espèces peu appétées et réagissant mal au pâturage. Leur dominance est l'indice de sols pauvres dans lesquels des carences minérales peuvent être à craindre. La repousse de saison sèche est toujours médiocre.

- Loudetia arundinacea, aves Tristochya sp. et des proportions variables des espèces citées au paragraphe précédent, colonise les sols sableux sur de grandes étendues, particulièrement sur les Plateaux de Carnot. La valeur du pâturage sera inversement proportionnelle à l'abondance du Loudetia dans le tapis herbacé.

- Loudefia sp. et Monocymbium ceresiforme forment l'essentiel de la végétation sur les plateaux sableux au nord de Salo ef dans les clairières de savane situées autour de Berbérati. Ce sont des savanes très peu arbustives, à tapis herbacé bas et clairsemé. Le Loudetia, associé à des proportions variables d'Audropogon shirensis forme une strate supérieure d'environ $1 \mathrm{~m}$ de haut sous laquelle pousse le Monocymbium. Contrairement au Loudetia, cette dernière espèce est bien appétée mais avec un développement faible et une productivité très réduite. De tels pâturages ne pourront supporter qu'une faible charge. Il sera bon de procéder à des dosages minéraux et d'oligoéléments afin de parer à d'éventuelles carences.

Loudetia sp. se rencontre en proportions variables dans la plupart des savanes.

c) Sovanes sur sols latéritiques et végétation sur cuirasses et dalles rochcuscs.

Ce sont encore différentes espèces du genre Loudetia qui dominent dans les sols comportant de fortes proportions de gravillons ferrugineux ou une cuirasse à faible profondeur. Ces espèces sont peu ou pas appétées. Etant donné le sol qui les porte, leur productivité est réduite : elle est pratiquement nulle en saison sèche.

Loudetia simplex occupe les sols renfermant les plus fortes proportions d'éléments concrétionnés, par exemple sur des collines au nord de Niem.

Loutetia arundinacea domine dans les sols gravillonnaires, par exemple sur le plateau de Bokolobo près de Bambari et au nord de Niem, associé alors avec Loudetia kagerensis.

En fonction de la teneur en gravillons ou de la profondeur de la cuirusse, d'autres graminées, Andropogonnées ou Panicées peuvent accompagner les Loudetia et valoriser ainsi le pâturage.

D'autres espèces de Loudetia, totalement inappétées, ainsi que Brachiaria reticulata (dans la région de Bambari), Sporobulus festivus occupent les affleurements de cuirasse.

Sur les dalles granitiques, particulièrement dans la région de Bocaranga on rencontre Hyparrhenia of. notolasia, susceptible de donner un bon pâturage de saison des pluies, très appété.

B. - Peuplements herbacés de vallée, sur sols humides ou plus ou moins inondables.

On peut distinguer differents types de végétation :

- alluvions inondées en saison des pluies. Prairie flottante d'Echinochloa stagnina exploitable en saison sèche (Bourgou). C'est un excellent pâturage, mais peu répandu.

- zones alluviales sableuses temporaire- 
ment inondables; peuplement à peu près pur de Pennisefum purpereum (herbe à éléphants). Bon pâturage d'appoint pour la saison sèche, mais n'existe que sur des superficies limitées $(P$. purpureum se rencontre aussi dans la végétation des jachères).

- zones tourbeuses engorgées de vallée. Peuplament herbacé variable toujours riche en Cypéracées; pâturage très médiocre sinon de valeur totalement nulle. On nous a signalé cependant un Scleria (Label Kalassa en Borroro) recherché comme fourrage de saison sèche.

II s'agit donc de zones de superficies réduites mais susceptibles cependant de fournir dans certains cas un appoint très précieux pour la saison sèche.

\section{$2^{\circ}$ La végéfation secondaire des jachères.}

Des superficies considérables de forêt ou de savane ont été défrichées à des fins culturales, à des dates plus ou moins récentes. II en résulte toujours une modification profonde de la végétation qui demande de nombreuses années pour s'effacer.

\section{a) Zones préforestières.}

Des savanes secondaires probablement récentes, formées d'un peuplement sub-pur de Pennisetum purpureum occupent une bande de largeur inégale au nord de la forêt, dans la région de Bangui par exemple, ou à Dario, au S. O. de Berbérati.

Ce sont de bons pâturages particulièrement pour la saison sèche, mais des déséquilibres minéraux peuvent se manifester, comme à Bangui, où l'on a trouvé dans le $P$. purpureum de forts excès de potassium qu'il faut compenser dans la ration par un apport de sodium.

b) Jachères récentes en zone forestière.

Ces zones peuvent présenter de l'intérêt pour les élevages sédentaires lorsque leur végétation comporte des proportions suffisantes de graminées. Les espèces les plus fréquentes sont, parmi les pérennes: Pennisetum purpureum, Panicum maximum, Beckeropsis unisefas et, parmi les annuelles:

\section{Pennisetum polystachyon, P. subangustum, Rottboellio exaltata.}

Ce sont de bonnes espèces fourragères mais qui sont souvent associées à des graminées forestières telles que Setaria megaphylla. Centotheca ou
Oplismenus sp., totalement dépourvues d'intérêt.

Ce type de végétation présente l'avantage de garder une productivité constante à peu près toute l'année du fait du climat de la zone forestière.

c) Jachères culturales en savane.

Lorsqu'elles ne sont pas envahies par des mauvaises herbes non graminéennes, elles peuvent donner des pâturages valables, mais soumis à certaines conditions d'exploitation en fonction de leur composition botanique. Différentes espèces peuvent constituer le peuplement graminéen :

- Imperafa cylindrica espèce pérenne donne un pâturage utilisable toute l'année, bien appété, mais uniquement sous forme de repousses très jeunes.

- Rottboellia exaltata, Pennisetum polystachyon, $P$. subangustum, Rhynchelytrum roseum, souvent associées, sont des espèces annuelles, donc utilisables uniquement en saison des pluies.

- Panicum phragmitoides, Hyparrhenia gracilescens, $H$. confinis, se développent souvent dans les jachères plus anciennes.

La première de ces espèces est très répandue. C'est une plante mal appétée qui se développe en fortes touffes, souvent associée heureusement à Brachiaria brizantha, plante bien appétée et réagissant bien au pâturage.

Ces jachères à Panicum paraissent occuper des superficies importantes en R. C. A., partout où la densité de la population est assez forte.

Hyparrhenia gracilescens est une espèce annuelle qui envahit parfois les jachères sur terrain sableux, sur les plateaux de Carnot en particulier. Elle est bien appétée.

Hyparrhania confinis se rencontre sur des terrains plus argileux; c'est une espèce de petite taille bien appétée et réagissont très bien au pâturage en formant un tapis dense et continu. Cette espèce constitue une grande partie des pĉturages de la station de Bossembélé.

\section{C) Pédologie ef valeurs fourragères}

\section{Pédologie.}

A titre indicatif, on trouvera ici quelques descriptions de profils et résultats d'analyses concernant les sols pris dans les différentes régions envisagées :

1. Station d'Elevage de Bouar. Profil El 2. Sol 
ferrugineux tropical, ligne de crête sous herbage dégradé :

- de 0 à $10 \mathrm{~cm}$, gris clair, argilo-sableux.

$-10-50 \mathrm{~cm}$ : beige, argileux, riches en sables grossiers.

- 50-150 cm : ocre, argileux, avec quelques micas.

Frofil issu de la décomposition du granite, lessivage ef concrétionnement faible ; $\mathrm{pH}$ assez cacide $(4,2$ à 4,5$)$. plus que sous savane intacte ( 5,5 à 6). Teneurs en chaux et magnésie plus faibles que sous savane, mais taux de potasse plus élevés.

Prélèvements : $1(0-5 \mathrm{~cm})-2(20-30 \mathrm{~cm})-$ $3(150 \mathrm{~cm})$.

2. Station d'élevage de Bossembélé, flanc de colline, au sud-ovesi de la ferme, zone prévue en extension. Sol assez argileux, assez bonnes teneurs en bases en surface, $\mathrm{pH}$ supérieur à 5 .

Prélèvements : $1(0-5)-2(25-35 \mathrm{~cm})-3$ $(150 \mathrm{~cm})$.

3. Plateaux de Salo, partie centrale sableuse à savane basse (essais de caféiculture). Sol très sableux. Teneurs en bases échangeables ef matière organique très faibles. Carences probables.

Prélèvements : $1(0-5 \mathrm{~cm})-2(20-30 \mathrm{~cm})-$ $3(100 \mathrm{~cm})$.
4. Plateaux de Carnot. Profil 58, à l'Est de la Lobaye vers Zcorosoungou. Savane peu arbustive.

Lit de sables blancs grossiers en surface.

- 0-25 cm. Brun-noir, sableux, grossier.

- 25-70 cm. Ocre brun, sableux, cohésion faible.

-- $70-230 \mathrm{~cm}$. Ocre-rouge, sablo-argileux.

Tous les profils observés dans cette zone sont très semblables, avec prédominance presque toujours des sables grossiers, Légère augmentation de l'argile en profondeur. Acidité généralement faible, avec des $\mathrm{pH}$ supérieurs à 5 . Teneur en bases échangeables souvent satisfaisantes en surface (sup. à 1,5 méq.).

Le potassium est bien représenté, les teneurs en chaux moyennes, mais elles diminuent rapidement avec la profondeur. Equilibre Ca/Mg correct. Des dosages d'oligoéléments ont fait apparaitre des carences en $\mathrm{Zn}, \mathrm{Mo}, \mathrm{Cu}$ et $\mathrm{Mn}$.

Prélèvements : $1(0-15 \mathrm{~cm})-2(30-40 \mathrm{~cm})-3$ $(150 \mathrm{~cm})$.

5. Dario, plantalion Le Gorf. Sols argileux à Pennisetum purpureum (Prélèvement E).

Terres exceptionnellement lourdes, mais bien structurées, teneurs en base échangeables très correctes.

Prélèvements: $1(0-10 \mathrm{~cm})-2(40 \mathrm{~cm})-3$ $(100 \mathrm{~cm})$.

D'après d'autres analyses, $\mathrm{pH}$ voisin de 7 .

\begin{tabular}{|c|c|c|c|c|c|c|c|c|c|c|c|c|}
\hline \multirow{2}{*}{ Prél. } & \multicolumn{4}{|c|}{ Pour 100 de t. fine } & \multirow{2}{*}{$\mathrm{pH}$} & \multicolumn{5}{|c|}{ Bases échangeables méq. p. $100 \mathrm{~g}$} & \multirow{2}{*}{$\begin{array}{l}C \\
\%\end{array}$} & \multirow{2}{*}{$\begin{array}{c}N \\
m g 100 \mathrm{~g}\end{array}$} \\
\hline & Arg. & Lim. & S. fin & S. gros & & Somme & $\mathrm{CaO}$ & $\mathrm{MgO}$ & $\mathrm{K}^{2} \mathrm{O}$ & $\mathrm{NaO}$ & & \\
\hline $1-1$ & 47,7 & 8.4 & 13,6 & 28,4 & 4,6 & 1,40 & 0,73 & 0,32 & 0,30 & 0,05 & 2,02 & 90 \\
\hline $1-2$ & $50,0(1)$ & 7,4 &, 96 & 32,5 & 4,55 & 0,49 & 0,21 & 0,11 & 0,10 & 0,07 & 1,66 & 84 \\
\hline $1-3$ & $58,7(2)$ & 10,1 & 10,7 & 19,3 & 4,45 & 0,45 & 0,21 & 0,10 & 0,09 & 0,05 & 0,79 & 83 \\
\hline $2-1$ & & 12 & 21 & 10 & 5,35 & 1,92 & 1,26 & 0,48 & 0,18 & $\operatorname{tr}$ & 1,92 & 132 \\
\hline $2-2$ & 57 & 8 & 25 & 8 & 5,20 & 0,40 & 0,19 & 0,10 & 0,11 & - & 1,42 & 98 \\
\hline $2-3$ & 67 & 4 & 20 & 8 & 5,70 & 0,32 & 1,1 & 0,08 & 0,13 & - & 0,33 & 50 \\
\hline $3-1$ & 7 & 4 & 19 & 70 & 5,0 & 0,28 & 0,06 & 0,11 & 0,08 & 0,03 & 0,72 & 40 \\
\hline $3-2$ & 8 & 4 & 21 & 66 & 5,0 & 0,11 & tr & 0,02 & tr & 0,02 & 0,33 & 26 \\
\hline $3-3$ & 16 & 4 & 11 & 58 & 5,35 & 0,12 & tr & 0,10 & tr & 0,02 & 0,22 & 24 \\
\hline $4-1$ & 11,6 & 2,4 & 38,9 & 45,1 & 5,25 & 1,46 & 0,80 & 0,25 & 0,24 & 0,17 & 1,18 & 82 \\
\hline $4-7$ & 12,2 & 1,8 & 41,3 & 42,3 & 4,85 & 0,79 & 0,35 & 0.14 & 0,17 & 0,13 & 0,85 & 68 \\
\hline $4-3$ & 20,4 & 1,0 & 32,3 & 44,6 & 4,50 & 0,73 & 0,25 & 0,14 & 0,18 & 0,16 & 0,21 & 35 \\
\hline $5-1$ & 27,6 & 25.4 & 35,7 & 84 & - & 18,6 & 11,2 & - & - & - & 3,96 & 40 \\
\hline $5-2$ & 60,5 & 16,8 & 20,2 & 3,0 & - & 6,5 & 1,6 & - & - & - & 0,66 & 51 \\
\hline $5-3$ & 59,1 & 19,4 & 16,8 & 3,3 & - & 7,4 & 1,65 & - & - & - & 0,66 & 51 \\
\hline $6-1$ & 45 & - & - & $20^{12}$ & 5,5 & 5,1 & - & - & - & -" & 0,24 & 43 \\
\hline $6-2$ & 65 & - & - & 17 & - & 1,5 & - & '- & $\ldots$ & - & - & 180 \\
\hline & & & & & & & & & & & & \\
\hline
\end{tabular}


6. Besson, sol sur micaschistes, assez argileux, ocre, profond, bonne structure : $\mathrm{pH}$ supérieur à 5 . Teneurs en base échangeables correctes en surface. Déficit potassique.

Prélèvements : $1(0-15 \mathrm{~cm})-2(40-50 \mathrm{~cm})$.

\section{$2^{\circ}$ Analyses fourragères.}

On trouvera ci-dessous un certain nombre d'analyses fourragères, effectuées sur les échantillons suivants :

1. Besson, route Besson-Baboua, peu après le carrefour avec la piste de Godro (emplacement de la photographie). Zone régulièrement pâturée, prélèvement moyen ; tapis à dominance de Panicum phragmitoides, avec Hyparrhenia diplandra, Paspalum scrobiculatum, Arundinella sp., Digitaria polybotrya.

2. Besson, piste vers Mayo-Lora, après le passage de la première rivière. Prélèvement moyen, tapis herbacé assez dense, modérément pâturé : Hyparrhenia diplandra, Panicum phrogmitoides, Urelytrum incompletum dominants, strate inférieure assez dense à Paspalum scrobiculatum et Digitaria polybotrya.

$\mathrm{Ce}$ deuxième type de pâturage est moins apprécié que le premier: les bêtes en reviendraient, paraît-il, malades. Sa valeur fourragère globale est cependant un peu meilleure, mais les teneurs minérales plus faibles. Ces différences sont cependant faibles et il est probable qu'il faille chercher plutôt une explication sanitaire. Des prospections n'ont cependant pas permis de trouver là des glossines.

3. M'Bissa, près de Berbérati, terrain sableux près du lac de retenue. Pâturage à Panicum phragmitoides et Brachioria bizantha. Prélèvement de Brachiario.

4. Même localité, mais aux environs du parc de nuit des animaux. Terrains plus argileux, même type de végćtation, plus dense; prélèvement de Brachiaria.

Il y a relativement peu de différences entre ces deux prélèvements. Le terrain sablo-colluvial de bas-fonds, plus humide et plus humifère, fournit cependant un fourrage d'un peu meilleure qualité.
5. Même localité et même station que le prélèvement 3. Prélèvement de Ponicum.

6. Même endroit que le prélèvement 4. Prélèvement de Panicum. On notera que le Panicum a une valeur fourragère bien inférieure à celle du Brachiaria. L'analyse des deux prélèvements de cette espèce est très semblable.

7. Dario, près de Berbérati, plantation Le Goff. Pennisetum purpureum non pâturé. On notera les très fortes teneurs en matières protéiques et minérales ef le déséquilibre dû à un excès de potassium. Un tel fait avait déjà été observé dans du Pennisetum prélevé à Bangui.

8. Même endroit, Pennisetum purpureum, forme pâturée. Par rapport à la forme intacte, la valeur fourragère est un peu moindre, mais l'excès potassique est atténué.

9. Plateaux sableux de Salo, carrcfour de la route de Banyanga. Savane basse et clairsemée d̀ Loudetia sp. et Monocymbium ceresiiforme. Prélèvement de Loudetia sp.

10. Même station, prélèvement de Monocymbium.

La valeur de ces deux fourrages est faible. On notera en particulier les faibles teneurs en matières protéiques et une nette carence phosphorique, particulièrement chez le Monocymbium.

11. Nalimo; sur la route de Salo. Même type de savane, prélèvement de Loudetia sp.

12. Même station, prélèvement de Monocymbium.

On peut faire les mêmes remarques que cidessus. L'échantillon 12 est particulièrement pauvre.

13. Pâturages de Diomo; forme pâturée en tapis dense à forte dominance d'Elymandra androphila. Prélèvement de cette espèce. La valeur fourragère est assez médiocre.

16. Station de Bossembélé, pâturages au nord de la route, prélèvement d'Hyparrhenia confinis, forme pâturée.

17. Même localité, prélèvement de Brachiaria brizantha, forme pâturée.

Les analyses ont été effectuées par le Laboratoire d'Alimentation de l'Ecole Nationale Vétérinaire d'Alfort. Les teneurs sont données en 


\begin{tabular}{|c|c|c|c|c|c|c|c|c|c|c|c|}
\hline Echant. & $\begin{array}{c}\text { Humi- } \\
\text { dité }\end{array}$ & $\begin{array}{c}\text { Matière } \\
\text { sèche } \\
\text { tot. }\end{array}$ & $\begin{array}{l}\text { Matière } \\
\text { prot. } \\
\text { brute }\end{array}$ & $\begin{array}{c}\text { Cellulose } \\
\text { bruie } \\
\text { (Wcen } \\
\text { de) }\end{array}$ & $\begin{array}{l}\text { Matière } \\
\text { minér. }\end{array}$ & P & $\mathrm{Ca}$ & $\mathrm{Na}$ & K & $\begin{array}{l}\text { Matière } \\
\text { prot. } \\
\text { digest. } \\
\text { in vifro }\end{array}$ & \begin{tabular}{|c} 
U. F. \\
par'kg \\
de mat. \\
sèche
\end{tabular} \\
\hline 1 & 10,00 & 90,00 & 7,47 & 33,20 & 7,60 & 0,13 & 0,75 & 0,01 & 0,75 & 2,61 & 0,41 \\
\hline 2 & 9,00 & 91,00 & 9,18 & 30,1 & 5,64 & 0,12 & 0,55 & 0,01 & 0,70 & 3,4 & 0,52 \\
\hline 3 & 9,60 & 90,40 & 7,30 & 28,80 & 9,86 & 0,28 & 0,60 & 0,01 & 1,25 & 3,52 & 0,51 \\
\hline 4 & 10,50 & 89,50 & 5,20 & 33,60 & 5,28 & 0,12 & 0,55 & 0,01 & 0,70 & 7,37 & 0,42 \\
\hline 5 & 10,70 & 89,30 & 8,64 & 30,90 & 8,00 & 0,17 & 0,55 & 0,01 & 1,75 & 3,96 & 0,46 \\
\hline 6 & 9,70 & 90,30 & 6,30 & 34,10 & 5,36 & 0,12 & 0,40 & 0,01 & 0,8 & 2, & 0,42 \\
\hline 7 & 5,20 & 94,80 & 12,24 & 29,70 & 19,00 & 0,21 & 0,55 & 0,01 & 4,37 & 5,58 & 0,44 \\
\hline 8 & 10,80 & 89,20 & 7,38 & 33,30 & 11,80 & 0,11 & 0,55 & 0,01 & 2,20 & 3,06 & 0,39 \\
\hline 9 & 8,60 & 91,40 & 3,60 & 36,80 & 4,54 & 0,10 & 0,40 & 0,01 & 0,75 & 1.4 & 0,37 \\
\hline 10 & 11,30 & 88,70 & 3,20 & 34,00 & 4,05 & 0,08 & 0,55 & 0,01 & 0,45 & 1,2 & 0,40 \\
\hline 11 & 9,40 & 90,60 & 3,50 & 37,10 & 4,3 & 0,08 & 0,55 & 0,01 & 0,75 & 2,0 & 0,34 \\
\hline 12 & 9,90 & 90,10 & 2,90 & 35,4 & 4,35 & 0,07 & 0,60 & 0,01 & 0,50 & 0,92 & 0,37 \\
\hline 13 & 9,90 & 90,10 & 7,74 & 30,0 & 8,95 & 0,17 & 0,60 & 0,01 & 0,70 & 2,47 & 0,47 \\
\hline 16 & 9,90 & 90,10 & 6,30 & 30,40 & 8,45 & 0,22 & 0,60 & 0,01 & 1,00 & 2,70 & 0,48 \\
\hline 17 & 14,1 . & 85,9 & 6,75 & 34,0 & 6,80 & 0,15 & 0,45 & 0,01 & 1,00 & 2,79 & 0,46 \\
\hline
\end{tabular}

grammes pour 100. Les valeurs fourragères sont données sous toute réserve, compte tenu des méthodes officielles employées qui sont très critiquables.

Il est, en effet, très probable que les critères utilisés pour interpréter ces analyses, coefficients de digestibilité en particulier, établis pour des fourrages et des animaux européens, ne sont pas directement applicables en pays tropical.

Quelle est, en définitive, la valeur fourragère de ces différents prélèvements?

Lu valeur d'un foin en Europe est comprise en gros entre 0,33 et 0,60 . U. F. au kilo. A l'état $\mathrm{sec}$, les fourrages analysés ont donc une valeur convenable, les chiffres les plu's bas étant obtenus dans la région de Salo.

En ce qui concerne les matières proléiques digestibles, en faisant le rapport: mat. prot. digest./U. F. par $\mathrm{kg}$ de M. S., on obtient des valeurs comprises entre 12 (Pennisetum purpureum à Dario). et 2,5 (route de Salo). Sauf dans un cas, les chiffres sont inférieurs à 8 ce qui est un coefficient déjà généralement considéré comme faible. On a donc une déficience assez générale en azote, surtout dans la région de Salo.

Les teneurs en cellulose, voisines de 30, sont assez correctes.

Les proportions de $\mathrm{P}+\mathrm{Ca}+\mathrm{Na}+\mathrm{K}$ par rapport aux matières minérales totales sont faibles ef toujours inférieures au $1 / 3$, le reste étant donc constitué en grande partie par de la silice.

La carence est souvent assez accusée en phos- phore : les teneurs en cet élément atteignent rarement $0,2 \%$ et dans la région de Salo elles sont inférieures ou parfois égales à $0,1 \%$, ce qui est nettement insuffisant. Les teneurs en calcium ont une assez bonne valeur moyenne. Le rapport $\mathrm{Ca} / \mathrm{P}$, qui devrait normalement être compris entre 1 et 2 est toujours trop fort (supérieur à 6 dans la région de Salo), ce qui risque de provoquer des troubles dans l'assimilation. II y a parfois un excès assez fort en potassium.

$$
\text { * * * }
$$

En définitive, une analyse précise de la végétation est indispensable si l'on veut pouvoir suivre l'évolution de la végétation d'un pâturage ou en analyser exactement la valeur.

Dans l'immédiat, les éléments que nous avons donnés ci-dessus suffisent pour choisir un pâturage, déterminer entre plusieurs emplacements quel serait le meilleur, ou pour toute reconnaissance générale.

\section{II. ÉVOLUTION ET DÉGRADATION DE LA VÉGÉTATION DES PATURAGES}

\section{A) Les modes d'utilisation des pâturages}

Le problème du pâturage ne se pose guère en ce qui concerne le petit élevage sédentaire. II 


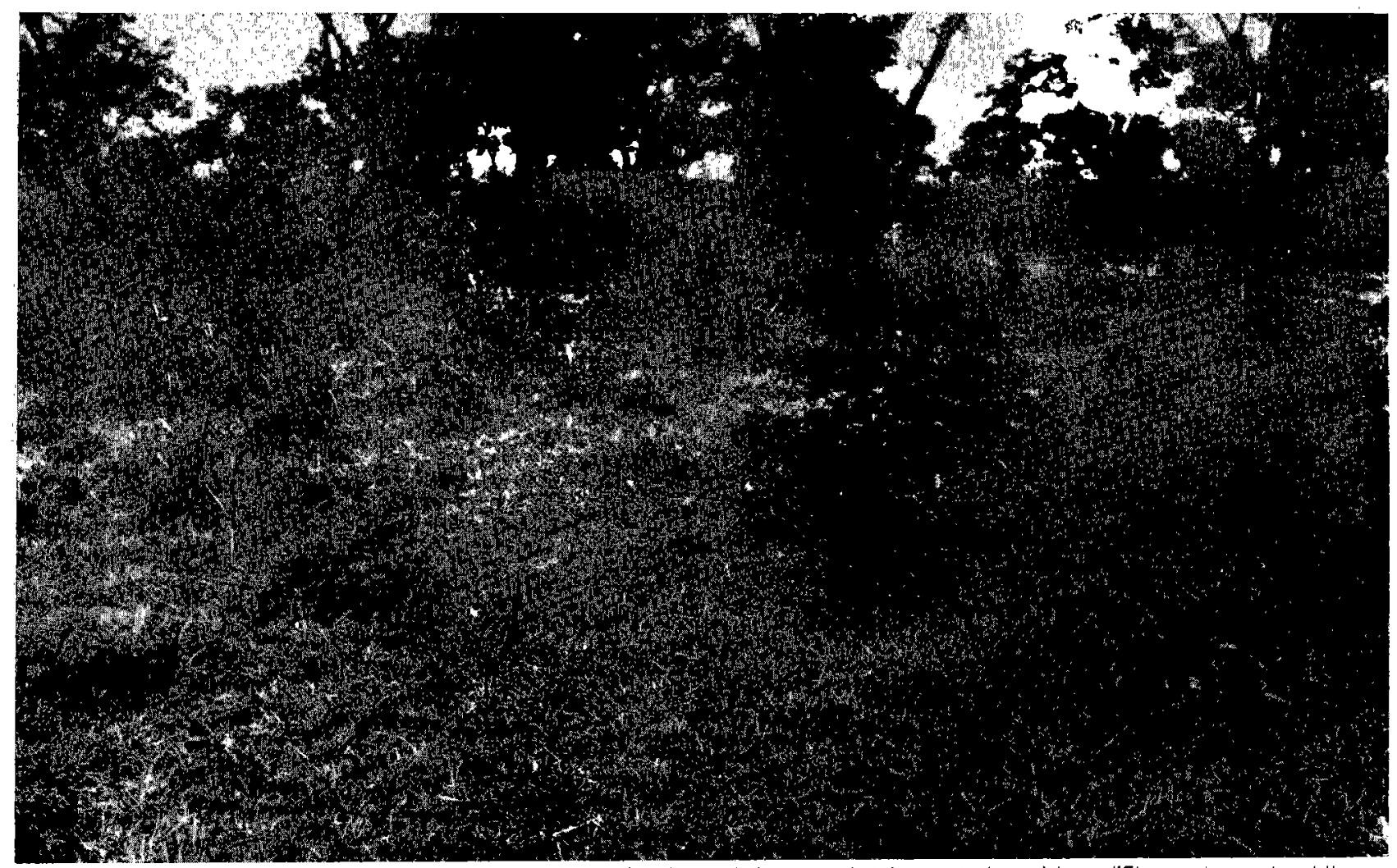

Djomo (Ardo Sani), à gauche de la route allant vers Djomo à la limite de la savane boisée : tapis dense à base d'Elymandra androphila. Les grosses touffes refusées sont du Panicum phragmitoides.

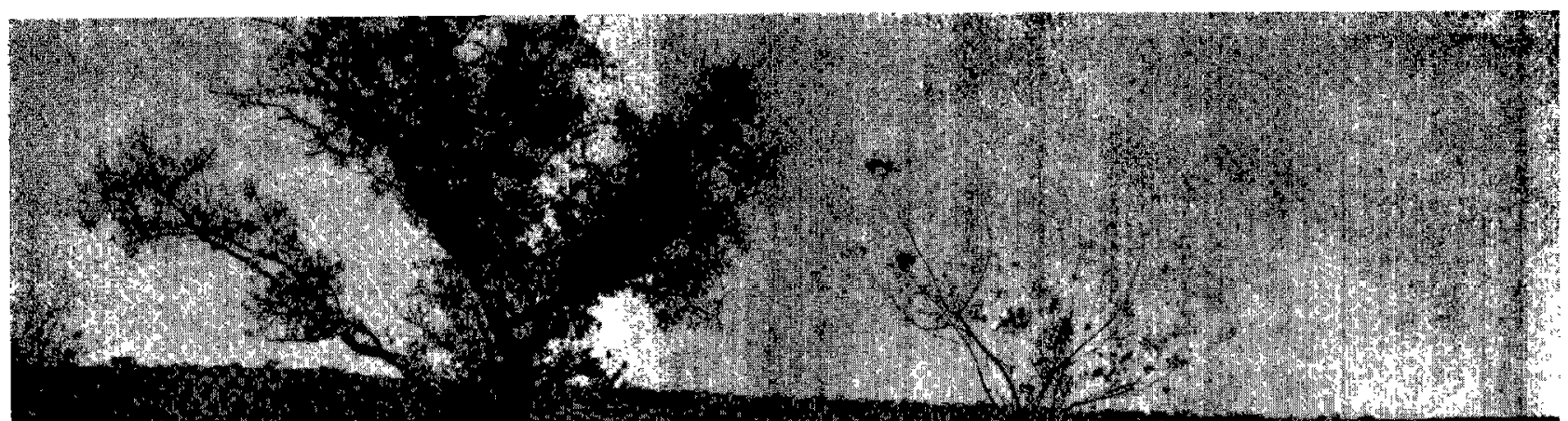

Djomo (Ardo Sani). Touffes de Panicum phragmitoides en refus. Petits individus de Parinari curatellaefolia en train de dépérir. 


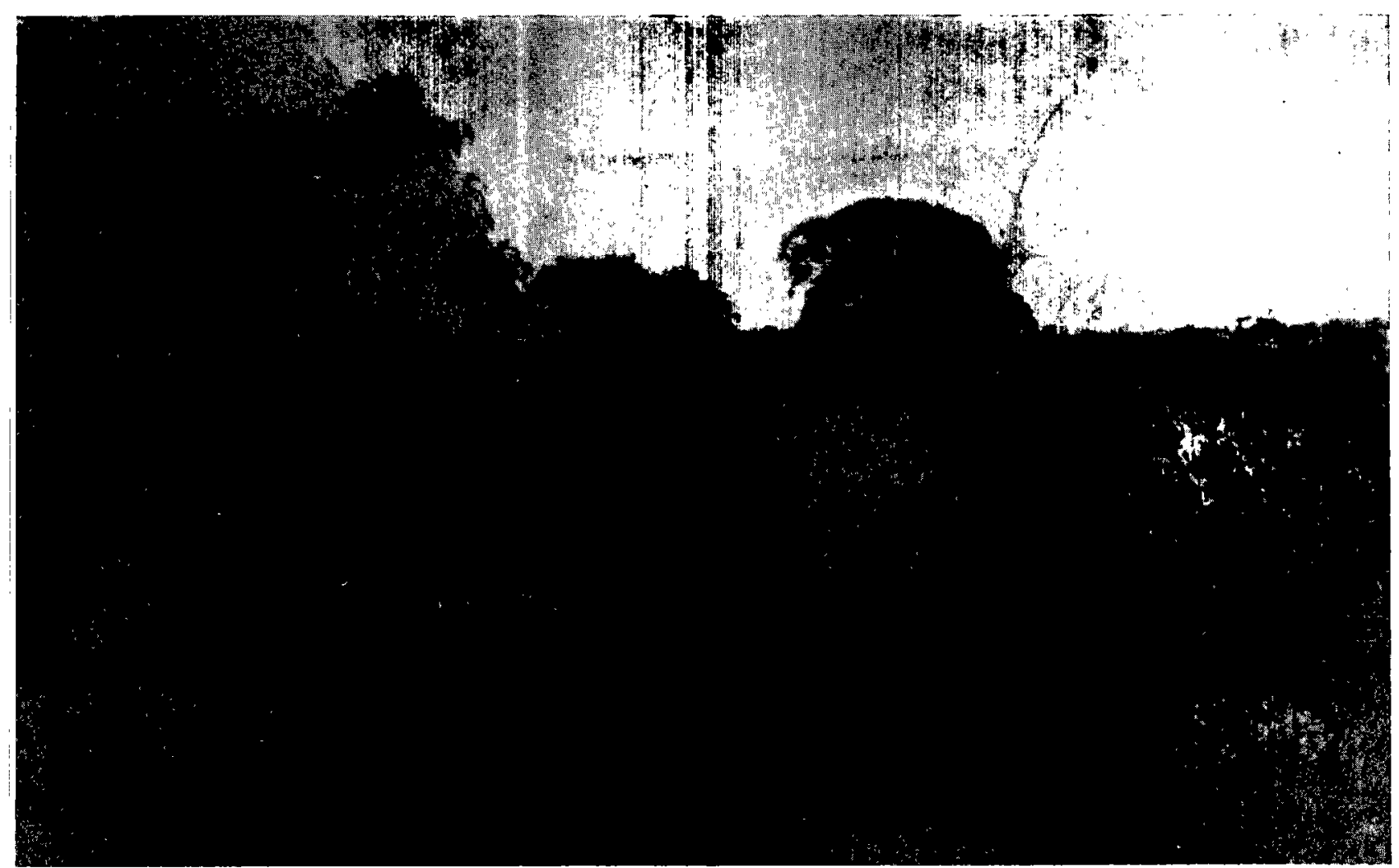

Sarki, centre de traitement d'Ardo-Badi, photographie prise en direction de la rivière depuis le coin gauche du Dippingtonk, côté sortie. Invosion du pâturage par Croton macrostachyus, très reconnaissable à son port en boule. Pałturage très dégradé et en grande partie dénudé.

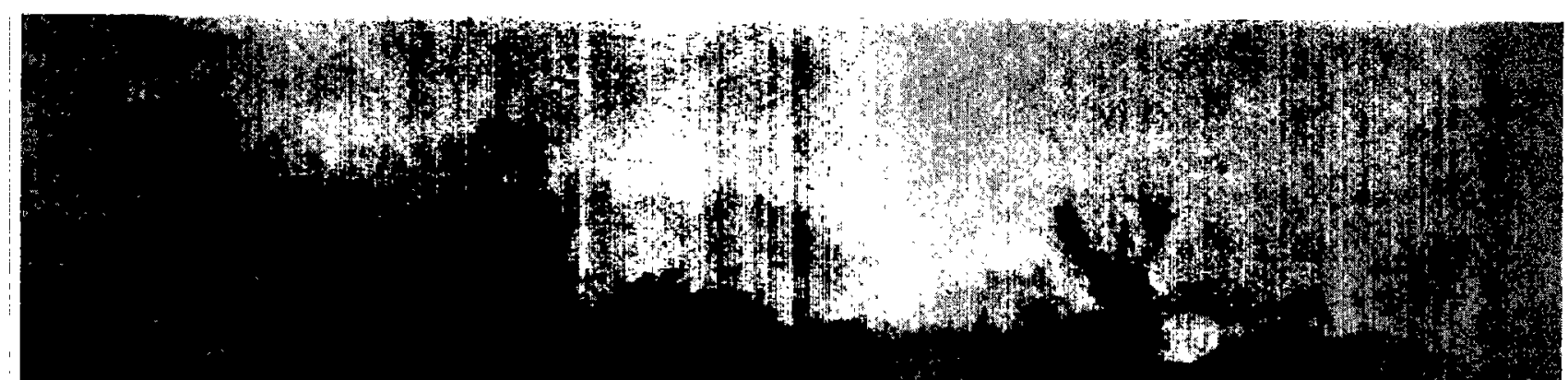

Ancien Yala, carrefour de la bretelle de Besson ovec lo route Bodo-Godro, à gauche de la route, en direction de Godro. II y a eu un poste à lait à cet endroit jusqu'en 1952. Le sol est resté ensuite pratiquement nu du fait de la présence constante de bétail ; invasion de Solanum torvum en 1954 ; n'a plus été páturé depuis. Les Solanum ont actuellement disparu et sont remplacés par un tapis très dense à base surtout d'Hyparrhenia diplandra. Aux alentours (arrière-plan), l'ancien páturage très dégradé est envahi par les Harungana. 


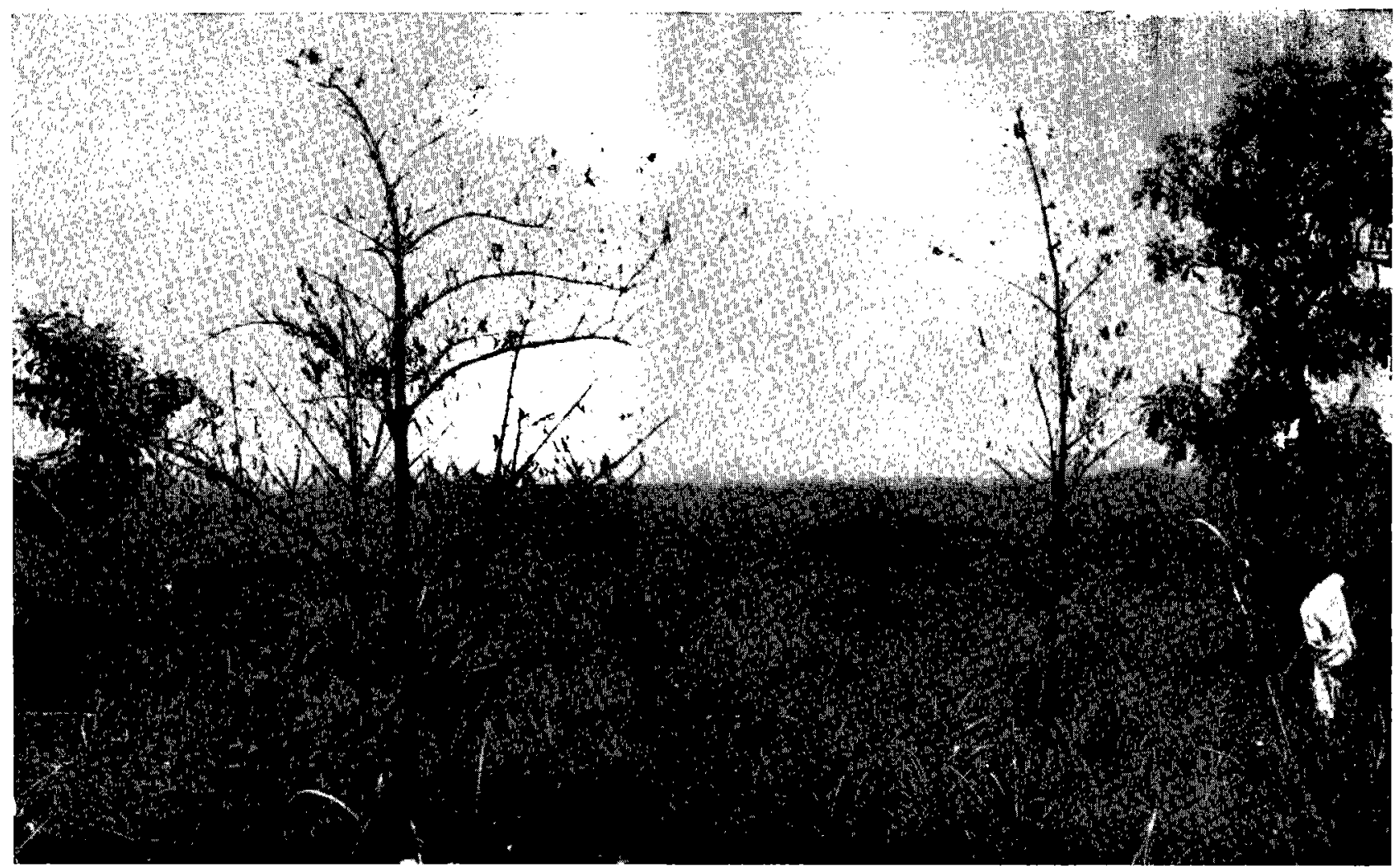

Cote 1.167 sur le raccourci joignont Besson à la route Godro-Bodo. Vue prise en direction de Mayo-Lara. Harungana à port élancé ayant été tués par le feu. Tapis graminéen assez dense d'espèces pérennes. Ancienne zone dénudée, au repos depuis environ 2 ans.

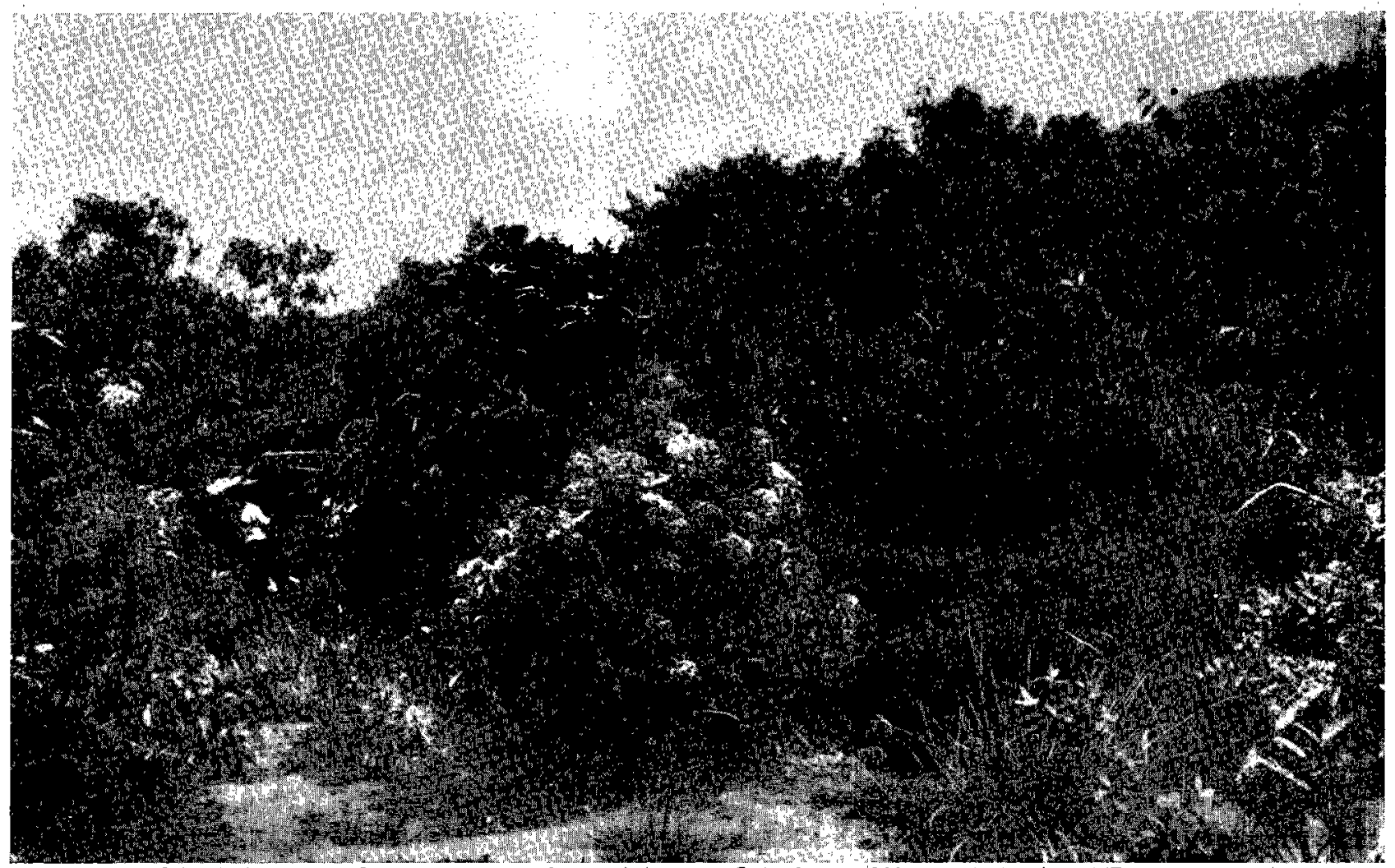

Près de Besson, - Raccourci joignant Besson à la route Godro-Besson, 300 m après la côte 1.167 vers Besson. Boisement secondaire d'Harungana madagascariensis. 
trouve une grande partie de sa subsistance dans les jachères autour des villages et ne cherche en savane qu'un complément. D'éventuelles carences alimentaires dans la végétation de cette dernière seront compensées par l'apport des plantes rudérales engraissées par tous les détritus du village. De fait, tous les troupeaux que nous avons pu voir étaient en excellent état, mêrne ceux placés sur des sols très pauvres comme sur les plateaux de Salo.

Enfin, du fait de la faible importance des troupeaux, la surcharge ou la dégradation des pâturages n'est pas à craindre.

Dans le cas des élevages nomades ou seminomades par contre, une exploitation rationnelle des pâturages est primordiale. La nécessité pour les animaux d'avoir constamment à leur disposition de l'herbe jeune implique la distinction entre pâturages de saison des pluies et de saison sèche. La croissance de l'herbe est en effet considérablement ralentie pendant la période sèche et les animaux auront besoin pendant celle-ci d'une superficie beaucoup plus importante.

Pendant la saison des pluies, les animaux ont tendance d̀ n'utiliser que le minimum de surface indispensable: assurés de voir une repousse jeune se renouveler rapidement, ils reviendront toujours aux mêmes endroits plutôt que d'aller chercher leur subsistance dans la savane plus ou moins intacte.

C'est ainsi que les périmètres utilisés par les troupeaux le sont souvent à $100 \%$, sans refus : ces zones seront impropres à assurer le pâturage de saison sèche. Celui-ci sera préparé par des brûlages qui suppriment les vieilles herbes et provoquent une jeune repousse.

Dans la région de Bovar-Bocaranga, on assiste à une véritable transhumance : les éleveurs se déplacent vers le sud, en direction des régions situées en contrebas du plateau de Bocaranga, et vers les grandes vallées de l'Ouham, de la Pendé et de la Nana où ils trouvent des pâturages d'appoint à $P$. purpureum ou $E$. stagnina.

A Carnot et dans le secteur de Bambari, les troupeaux sont plus stables et trouvent leur pâturage de saison sèche dans un périmètre réduit.

Les répercussions de ce mode d'exploitation sont considérables sur la végétation : les pâturages de saison des pluies sont presque toujours surexploités ; l'action nocive du piétinement est favorisée par les pluies et ceci explique que l'on assiste à des phénomènes de dégradation parfois spectaculaires qui seront décrits dans le paragraphe suivant.

Par contre, l'exploitation des pâturages de saison sèche est moins intense : la repousse étant faible, le rythme des rotations et la charge sont réduits, ainsi que les effets du piétinement. Enfin, sur ces zones, la végétation pourra se reconstituer pendant la saison des pluies.

Un premier remède pourrait donc être apporté au problème de la dégradation des pâturages par une meilleure répartition de l'exploitation dans le temps et dans l'espace. Si la date de début de la période d'utilisation des pâturages de saison sèche est impérativement fixée par les possibilités de brûlage et de circulation, il serait par contre souhaitable de prolonger leur exploitation pendant quelques semaines après le début des pluies, sans attendre cependant que l'herbe ait atteint un stade de croissance tel qu'elle devienne difficilement consommable.

\section{B) Evolution de la végétation dans les pâturages}

La mise en pâture d'une savane provoque dans la végétation des modifications importantes, mais finalement assez sembiables, quel' que soit le type d'origine de la végétation. Ces módifications se manifestent de différentes façons : évolution de la structure de la végétation et de la couverture du sol, changements dans la flore, bouleversement de l'équilibre entre les strates herbacées ef ligneuses. Leurs conséquences écoromiques peuvent être considérables.

1. Structure de lo végétation.

Dans les savanes soudano-guinéennes, la strate herbacée est essentiellement constituée par des grcminées cespiteuses pérennes, disposées en rouffes plus ou moins densément réparties sur le terrcin. Si ce tapis forme un couvert dense et difficilement pénétrable, le recouvrement réel au niveau du sol est en fait assez faible et de l'ordre généralement de $30 \%$. Cet aspect de la végétation apparaît nettement après le passage des feux, ou lorsqu'une savane vient d'être livrée au pôłurage. Les touffes sont peu nombreuses mais ce forte taille dans les cas des savanes sur sols profonds et frais, à Hyparrhenia diplandra, Andro- 

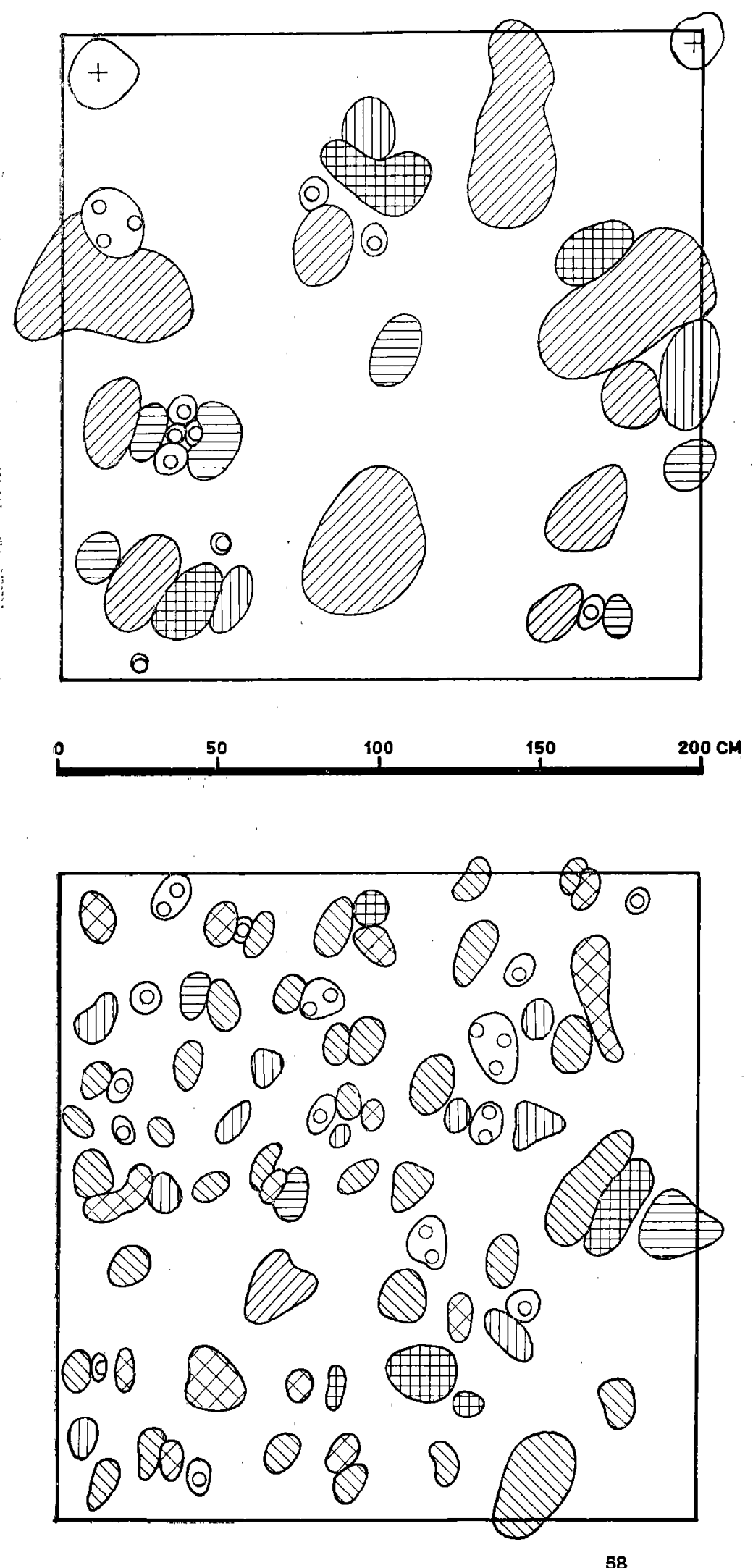

Fig. 2.

Savane ò Hyparrhenia Diplandra sur sols profonds.

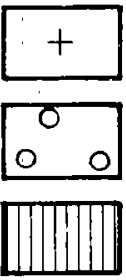

Schyzachyrium platyphyllum

Hyparrhenia diplandra

Panicum phragmitoides

Panicum fulgens

Andropogon pseudapricus

Hyparrhenia Lecomtei
Savane d. Hyparrhenia Lecomiei sur sols squelettiques. 


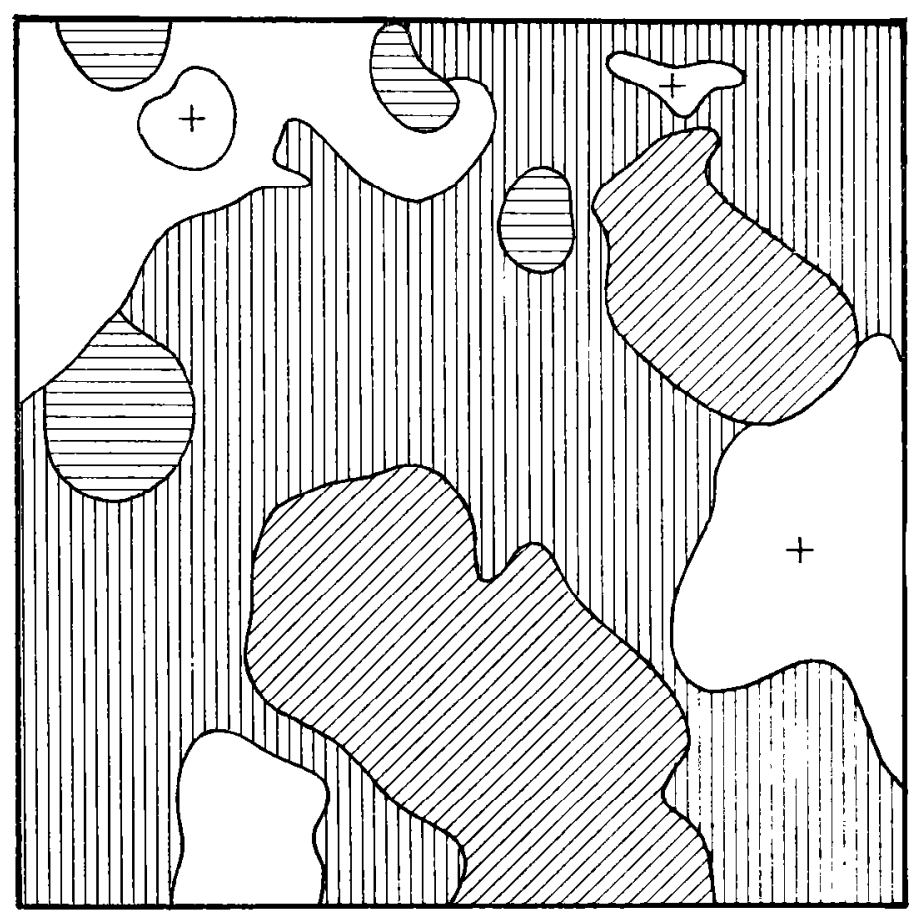

9

50

100

150

$200 \mathrm{CM}$

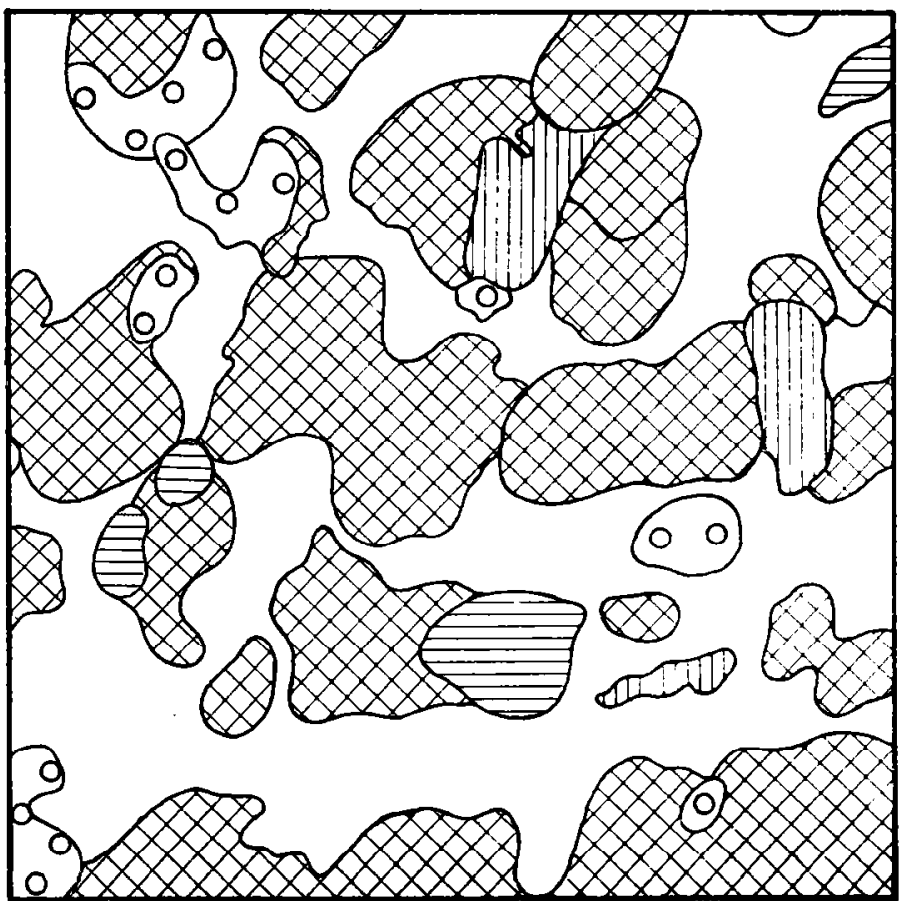

59
Fig. 3.

Savane d Hyparrhenia Diplandra sur sols profonds après un an de pâture.

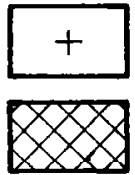

\section{Arbustes}

Hyparrhenia et Andropogon spp

\section{Panicum phragmitoides}

Schyzachyrium platyphyllum

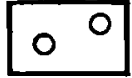

Bulbostylis spp.

\section{Hyparrhenia diplandra}

Sovane à Hyparrhenia Lecomtei sur sols squelettiques oprès un an de pâture. 
pogon gayanus ou Beckeropsis uniseta. Elles sont pius petites et plus nombreuses en terrain plus sec, par exemple, dans les savanes à Andropogon schirensis ou Loudetia arundinacea. A titre indicatif, les figures 2 et 3 (1) montrent le recouvrement du sol dans une savane vierge ef dans un pâturage.

L'effet du broutage et du piétinement, avec une charge de bétail et un rythme de rotation normal, provoque une modification et une amélioration profonde de cette structure : on assiste à une multiplication du nombre des pousses herbacées dans chaque touffe et à une augmentation de leur superficie par suite d'un étalement de la plante et d'un thallage plus vigoureux. Le coefficient de recouvrement au sol de 30 à $80 \%$ et parfois plus, et la densité des pousses herbeuses du simple au triple.

A ce stade, il n'y a pas de modifications notables de la flore; tout au plus peut-on constater un changement dans les proportions relatives des espèces : les plantes les plus appétées réagissent le plus vigoureusement et tendent à occuper le maximum de surface aux dépens d'espèces refusées qui gardent leur port cespiteux dressé. Ceci est très net dans le cas de pâturages qui, à côté de l'Hyparrhenia diplandra ou confinis, de l'Elymandra androphila ou du Brachiaria brizantha, comportent une certaine proportion de Panicum phragmitoides ou de Loudetia arundinacea.

II n'est ainsi pas rare de rencontrer de très beaux pâturages constitués par un tapis dense ef continu d'une herbe fine, régulièrement pâturée (Djomo près de Carnot avec Elymandra androphila, Sarki, près de Bocaranga, avec Hyparrhenia et Andropogon spp, Langandi près de Bambari avec Hyparrhenia diplandra).

Cette modification est donc très bénéfique et se traduit indubitablement par une amélioration de la qualité ef de la productivité du pâturage.

Mais cette amélioration est relativement instable et se dégrade rapidement en cas de surpâture. L'herbe disparaît ef laisse la place aux mauvaises herbes d'abord, puis à l'érosion.

(1) Fig. extr. de : Trochain et Koechlin. - Les pâturages naturels du sud de l'AEF, Bull. de l'I. E. C., no 15-16, 1958.

\section{Evolution de la végétation herbacée.}

Dès qu'un début de dégradation rend moins dense la couverture herbacée, des mauvaises herbes apparaissent:

a) II s'agit d'abord de graminées annuelles dont certaines sont appétées et ont une valeur fourragère correcte, en particulier une bonne teneur en matières azotées. Les principales espèces qui apparaissent sont les suivantes: Digitaria longiflora, D. polybotrya. Paspalum scrobiculatum, Eleusine indica. Dactyloctenium aegyptium, Eragrostis gangetica, Sporobolus pyramidalis, S. mollieri...

La prolifération de ces espèces, en plages plus ou moins étendues, est la conséquence non seulement d'une dénudation mais aussi d'une évolution du sol, superficiellement durci par le piétinement et enrichi par les déjections des animaux.

Les trois dernières des espèces citées ci-dessus ne sont pas appétées.

Toutes ces plantes sont de petite taille, disparaissent pratiquement pendant la saison sèche et ont une productivité faible : leur multiplication dans un pâturage diminuera donc considérablement sa capacité de charge.

b) Avec l'accentuation des phénomènes de dégradation, cette flore graminéenne sera à son tour remplacée par des plantes rudérales diverses dépourvucs de tout intérêt fourrager. On peut citer par exemple: Sida carpinifolia, Sida rhombifolia, Platystoma africana, Alternanthera sessilis, Amaranthus spinosus; A. viridis, Cassia tora, Acanthospermum hispidum, Spilanthes acmella. Chrysanthellum procumbens, Lconotis africana, Solanum torvum, S. aculeatissimum, Diodia scandens, etc..., etc.

Ces espèces apparaissent d'abord de façon diffuse mais arrivent à remplacer totalement la végétation graminéenne. Certaines forment des peuplements monospécifiques purs, comme Solanum Torvum qui a envahi les pâturages de la région de Baboua sur de grandes superficies.

A ce stade de dégradation, le pâturage a perdu toute valeur et doit être abandonné.

Sur des zones anciennement pâturées puis abandonnées se développent souvent dès peuplements de Panicum phragmitoides et d'Urelytrum incompletum. Ces deux espèces sont mal appétées, la seconde probablement à causè de son goût amer qui se transmettrait au lait. 


\section{Evolution de la végétation ligneuse.}

Dans les savanes naturelles, le feu de brousse maintient un équilibre pratiquement stable entre les strates herbacées et ligneuses. II n'en est plus de même dans les pâturages : si la charge est assez forte, le feu n'est plus possible ; les cîmes des arbustes vont s'étoffer, les basses branches et les rejets se développer. De plus, de nombreuses souches souterraines qui n'émettent normalement que quelques pousses aussitôt détruites par le feu, vont former de nouveaux arbustes. C'est ainsi que des essences comme Annoma arenaria, Burkea africana. Hymenocardia acida rejettent très vigoureusement. D'autres plantes ligneuses s'installent : par exemple une Légumineuse en forme de baguettes de 1 à 3 mètres de haut. Adenodolichos paniculatus, qui arrive à former d'importants buissons sur les sols d'origine granitique, à Bovar et à Bocaranga Certaines essences forestières normalement éliminées par le feu vont aussi pouvoir coloniser les plages dénudées: il s'agit surtout d'Harungana madagascariensis, et plus rarement de Croton macrostochyus.

Parallèlement à la dégradation du tapis herbacé on assiste donc à un embuissonnement du pâturage qui en réduit la surface utilisable ef favorise encore le développement des mauvaises herbes, généralement moins sensibles à l'ombre que les graminées. L'invasion par les espèces forestières est particulièrement grave. Elles prennent en effet un port buissonnant assez dense pour éliminer toute végétation herbacée savanicole : celle-ci est remplacée par des espèces forestières comme Desmodium adscendens, Setoria plicatilis, Leea guineensis, etc... On voit également s'installer des semis d'arbres forestiers, Fagara macrophylla, Trema guineensis, Allophyllus africanus, Alchornea cordifolia, Canthium venosum. C'est donc une véritable forêt qui remplace la savane. Insensible au feu, elle est inutilisable pour l'élevage.

A noter que les racines de Desmodium adscendens sont très riches en nodosités; le défrichemont dc ces zones secondairement boisées pourrait donc être intéressant pour l'agriculture.

L'importance des phénomènes d'embuissonnement ou de boisement est très variable selon les régions: dans la région de Carnot, il ne paraît pas y avoir actuellement d'extension mar- quée de la végétation ligneuse. A Bambari, nous avions noté un embuissonnement important par les essences de la savane, mais pas par des essences forestières. C'est dans le secteur de Bouar que ces phénomènes sontles plus marqués: la prolifération des rejets de Sphenostylis, d'Annona ou de Burkea est souvent considérable. Dans la région de Baboua (Godro, Besson) des superficies importantes ont été perdues pour l'élevage du fait de la prolifération de l'Harungana. Autour de Sarki et à Bouar, le Croton macrostachyus semble également, se montrer envahissant.

\section{C) Conservation et régénération des pâturages}

Cette question a été suivie de près dans la région de Bouar par le Dr Desrotour, actuellement Chef du Service de l'Elevage en R. C. A. Nous-mêmes l'avions déjà abordée lors de nos précédentes missions.

Il est d'abord indispensable de bien connaître quelle peut être l'évolution naturelle des différents types de végétation lorsque les pâturages sont plus ou moins chargés ou, au contraire mis au repos. On pourra seulement ensuite envisager quels seraient les moyens propres à modifier le cours de cette évolution dans un sens favorable.

10 Régénération de la végétation herbacée.

D'après ce que nous avons pu voir, un pâturage exploité avec une charge convenable est susceptible de se maintenir en bon état pendant de nombreuses années. Mais :

a) La mise en défens de zones peu dégradées ef ayant seulement subi un début d'invasion par des graminées annuelles semble suffire pour permettre la reconstitution rapide du peuplement d'origine.

b) Dans les stades suivants, le devenir de la végétation est plus incertain et dépend de l'état d'évolution du sol et de la nature des plantes présentes et susceptibles d'effectuer l'ensemencement :

- La mise en défens de terrains relativement peu dégradés semble aboutir à un peuplement de Panicum phrogmitoides. C'est un pâturage médiocre qui n'évoluera que lentement pour revenir aux stades primitifs à Andropogonnées. 
- Sur des sols très dégradés, on assiste souvent après le départ du bétail à une invasion de plantes non graminéennes. Cassia tora, Acanthospermum hispidum, Solanum torvum par exemple. Cette dernière espèce avait ainsi envahi des surfaces importantes dans la région de Baboua. Après deux ans environ, les Solanées ont dépéri et ont été remplacées par un tapis de graminées annuelles qui s'est peu à peu enrichi en Andropogonnées : un'bon pâturage s'est ainsi réformé.

- Sur sols dénudés, s'ils ont été décapés par l'érosion, ce sont des graminées annuelles qui s'installent (Sporobulus, Digitaria...) sur les accumulations de sol meuble formées derrière de microbarrages naturels.

- Sur les sols dénudés par piétinement et enrichis par les déjections, ce sont au contraire des plantes rudérales qui jovent le rôle de pionniers, les graminées ne venant qu'ensuite, annuelles d'abord, puis Panicum phragmitoides et espèces de savane.

Les phénomènes d'érosion, parfois importants, ne semblent cependant pas devoir prendre de développement catastrophique, comme c'est le cas, par exemple, à Madagascar. Une protection totale assurée pendant quelques années doit permettre au tapis végétal de se reconstituer.

Par ailleurs, des essais de réensemencement ont été effectués par le Docteur Desrotour. Les résultats n'ont, malheureusement, pas pu être suivis très exactement. Du Pennisetum subangustum a élé semé sur une surface surpaturée et très dénudée. II semble que ce semis ait permis d'éviter l'envahissement par des mauvaises herbes non graminéennes, puis l'embuissonnement du terrain. Le peuplement herbacé formé s'esł ensuite lurgement enrichi en graminées pérennes de bonne qualité. L'étude de cette lechnique mériterait donc d'être approfondie.

\section{Lutfe contre l'embuissonnement.}

Dans le cas de la végétation herbacée, une simple mise en défens permet souvent la régénération du pâturage après un laps de temps plus ou moins long. Le problème est malheureusement presque toujours compliqué par la prolifération de la végétation ligneuse. Si le tapis graminéen n'est pas trop dégradé, un ou deux ans de mise en défens suffiront pour pouvoir brôler à nouveau ef ramener la végétation ligneuse à ses proportions normales. II n'en sera plus de même si le pâturage est envahi par des graminées annuelles ou des rudérales incapables de fournir au feu un aliment suffisant.

L'intervention contre l'embuissonnement doit donc se faire très précocement, sinon il faudra avoir recours à de couteux moyens d'éradications chimiques ou mécaniques.

Tout ceci reste valable en ce qui concerne le développement des essences forestières (Harungana, Croton). Ces plantes, très sensibles au feu, seront facilement détruites par un incendie allumé au début de la période d'invasion, ce qui est souvent possible. Au début dc leur développement, au-dessus d'une strate herbacée encore assez fournie, ils prennent, en effet, un port élancé et l'ombrage est assez léger pour ne pas entraver le développement des graminées à leur pied.

$\mathrm{Si}$, au contraire, le sol est en partie dénudé ou recouverf d'un tapis bas, ces arbres prennent une forme plus dense, avec des branches basses et éliminent la végétation savanicole. Le feu ne sera plus possible et l'emboisement est alors irréversible.

L'exploitation des paturages de la R.C. A. soulève donc des problèmes importants. Les dégradations que nous avons pu constater se manifestent sur de grandes surfaces et provoquent une sérieuse baisséde la production. De plus, la dégradation de la végétation s'accompagne souvent d'une accentuation des phénomènes d'érosion. Ceux-ci ont fait l'objet d'une étude de MM. Benoît Janin et Roche, pédologues.

Dans l'état actuel des choses, où la surface des pâturages est limitée pour des raisons sanitaires, ces phénomènes ne peuvent aller qu'en s'amplifiant de plus en plus rapidement. $\|$ est donc urgent d'intervenir et d'envisager par quels moyens il serait possible d'enrayer la dégradation et même de régénérer les zones abîmées. Un programme d'action devra être mis au point afin d'amener les éleveurs à pratiquer un système plus rationnel. L'étude de ces questions fera l'objet du paragraphe suivant.

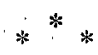




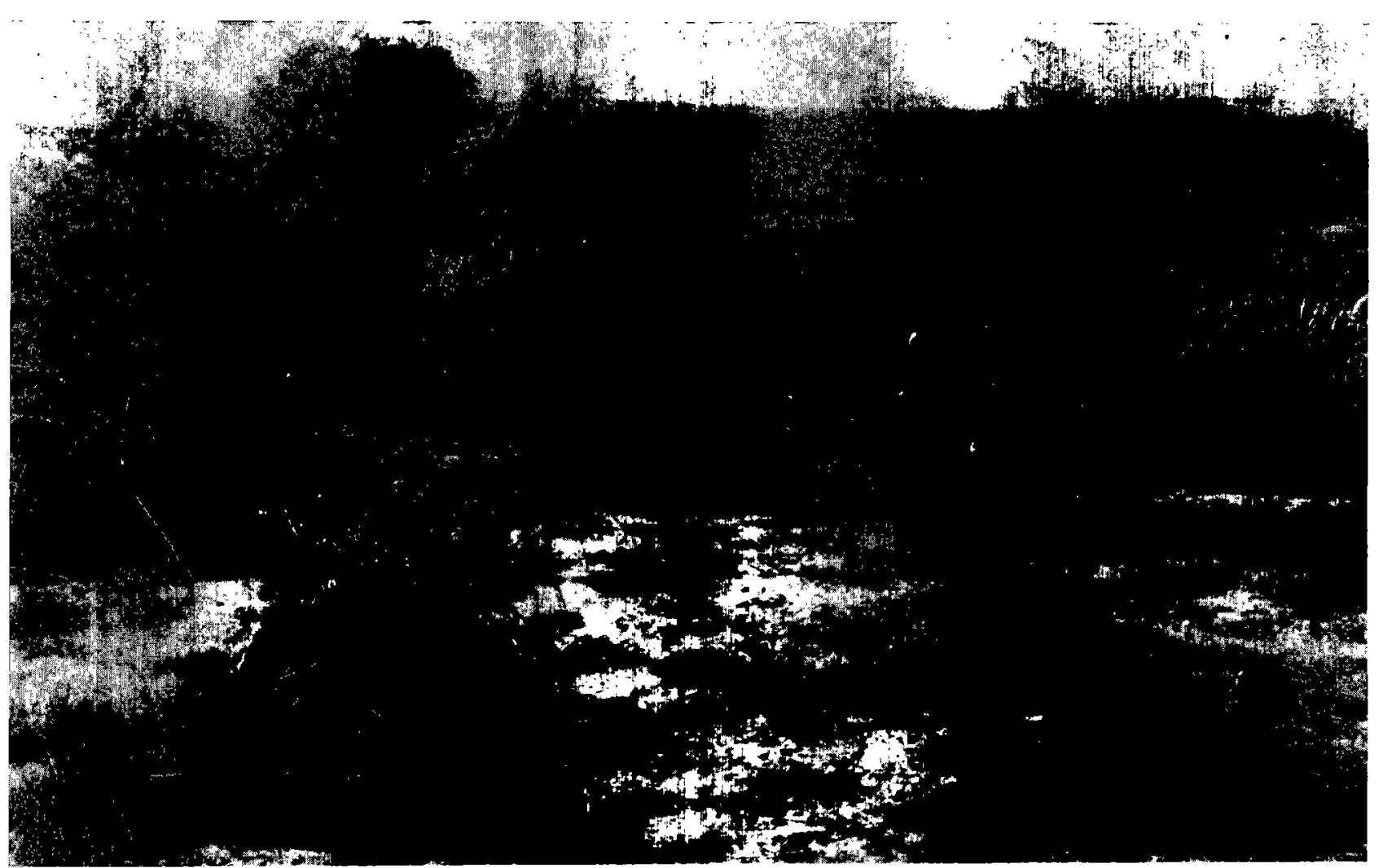

Raccourci joignant Besson à la route Godro-Bodo. $100 \mathrm{~m}$ adrès lo côte 1.167, vers Besson, essai de fossés anti-érosifs.

Sol érodé nu à part quelques touffes d'annuelles et quelques arbustes. Paspalum scrobiculatum planté sur les' talus des fossés.

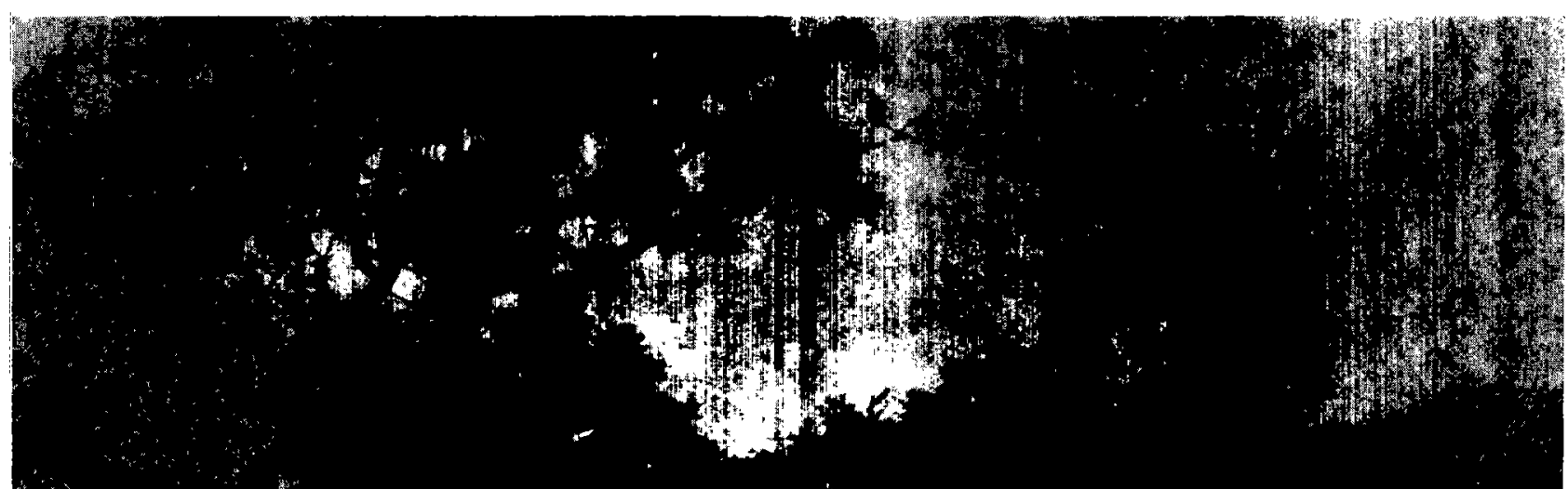

Route Besson-Baboua. $1 \mathrm{~km}$ après le carrefour avec la route de Godro. Pâturage en cours d'utilisation : sol partiellement dénudé et plages à bose de Paspalum scrobiculatum et Panicum phragmitoides. A gauche, couché sur le sol, un Harungana détruit par le feu. 


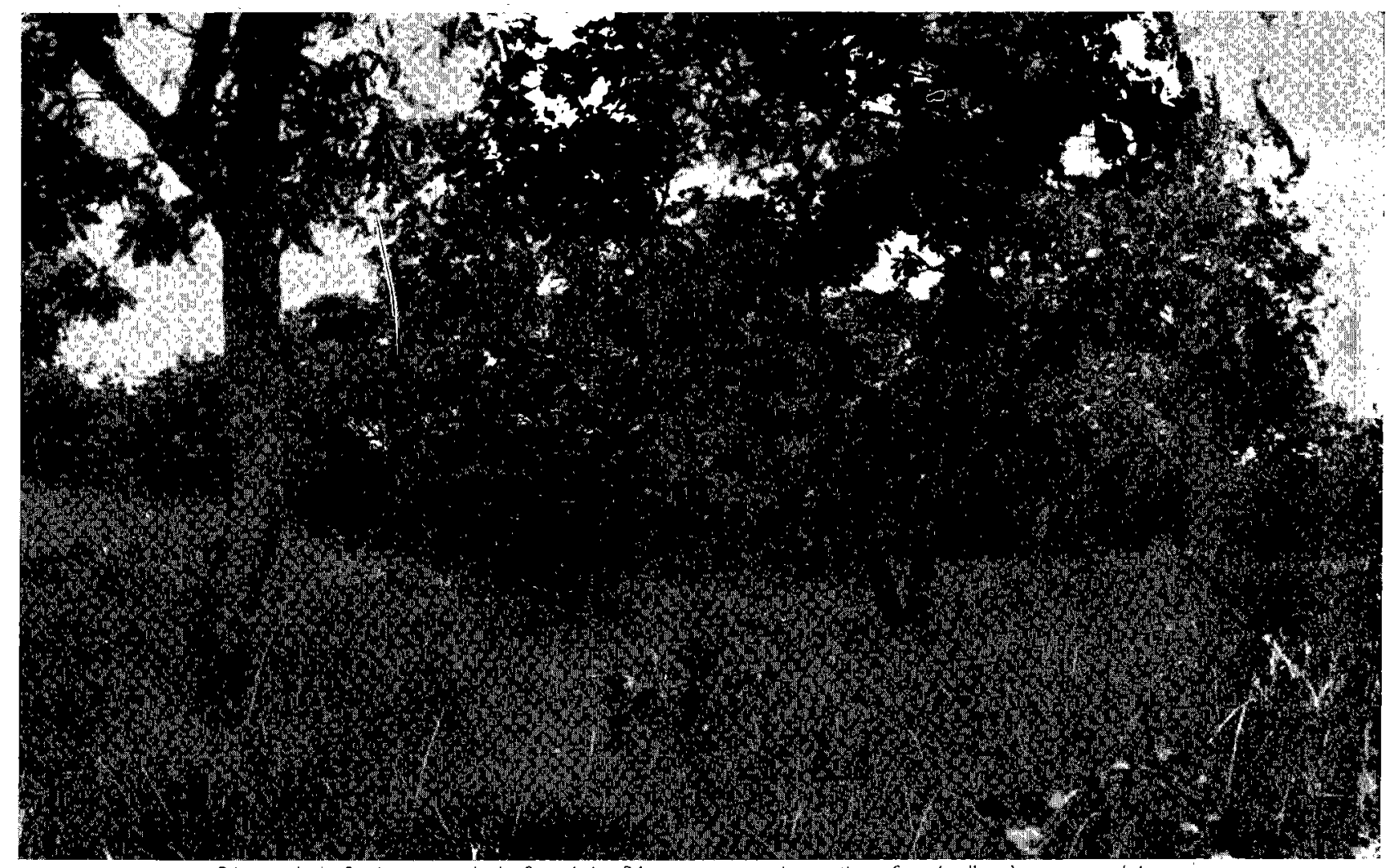

Djomo, Ardo Sani, autour de la Sangéré ; Pâluraye ras mais coninu formé d'espèces secondaires :

Panicum phragmitoides ef surtaut Paspalum scrobiculatum ef Eleusine Indica.

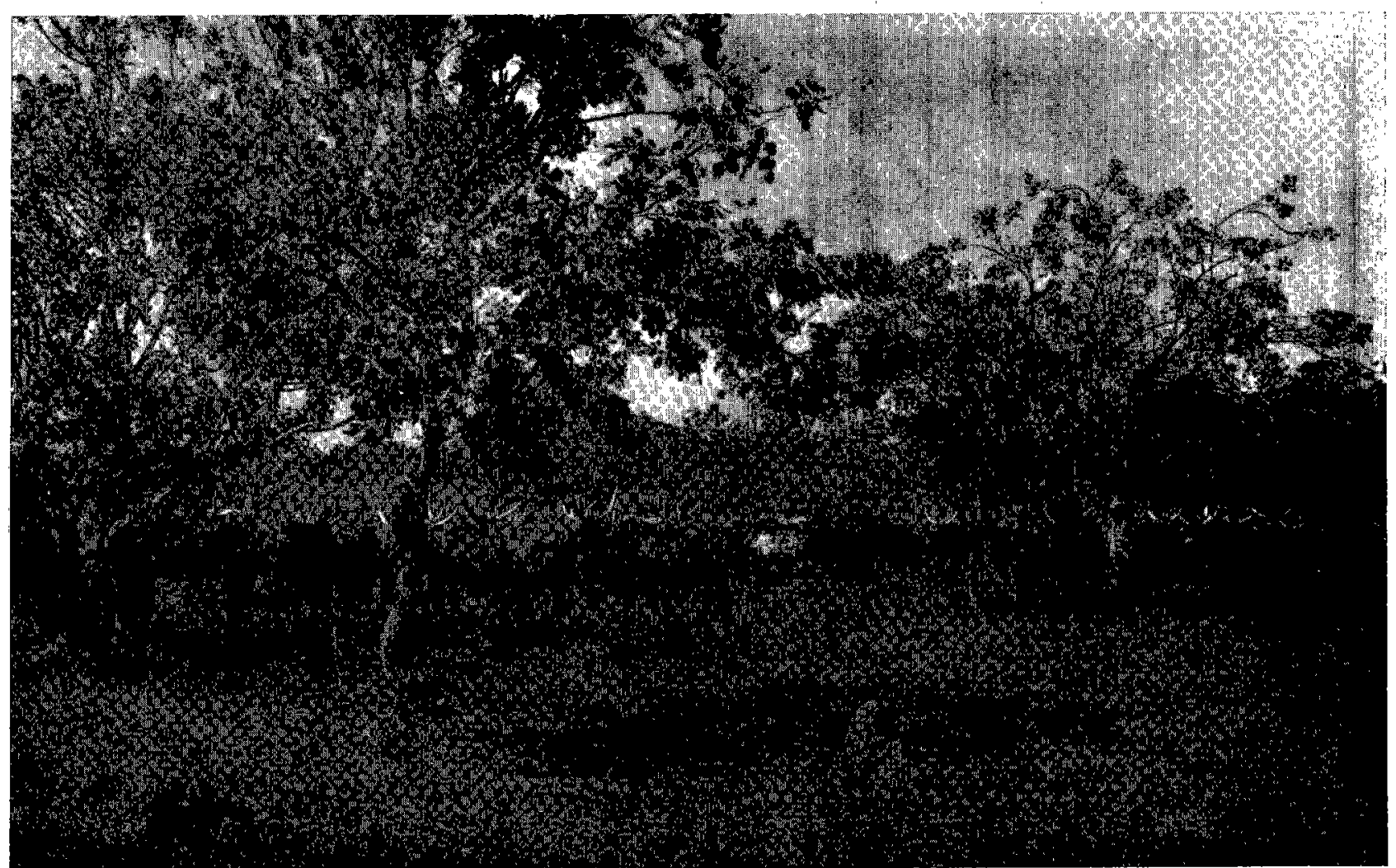

Carrefour de la route Besson-Tayo Lara avec la bretelle vers l'ancien Yala. Sur la bretelle, à 50 m du carrefour savane moyennement arbustive avec quelques Harungana. Zone dénudée jusqu'en 1954 puis pôturée en saison sèche uniquement. Panicum phragmitoides et Hyparrhenia (diplandra?) dominants, en tapis assez dense. 


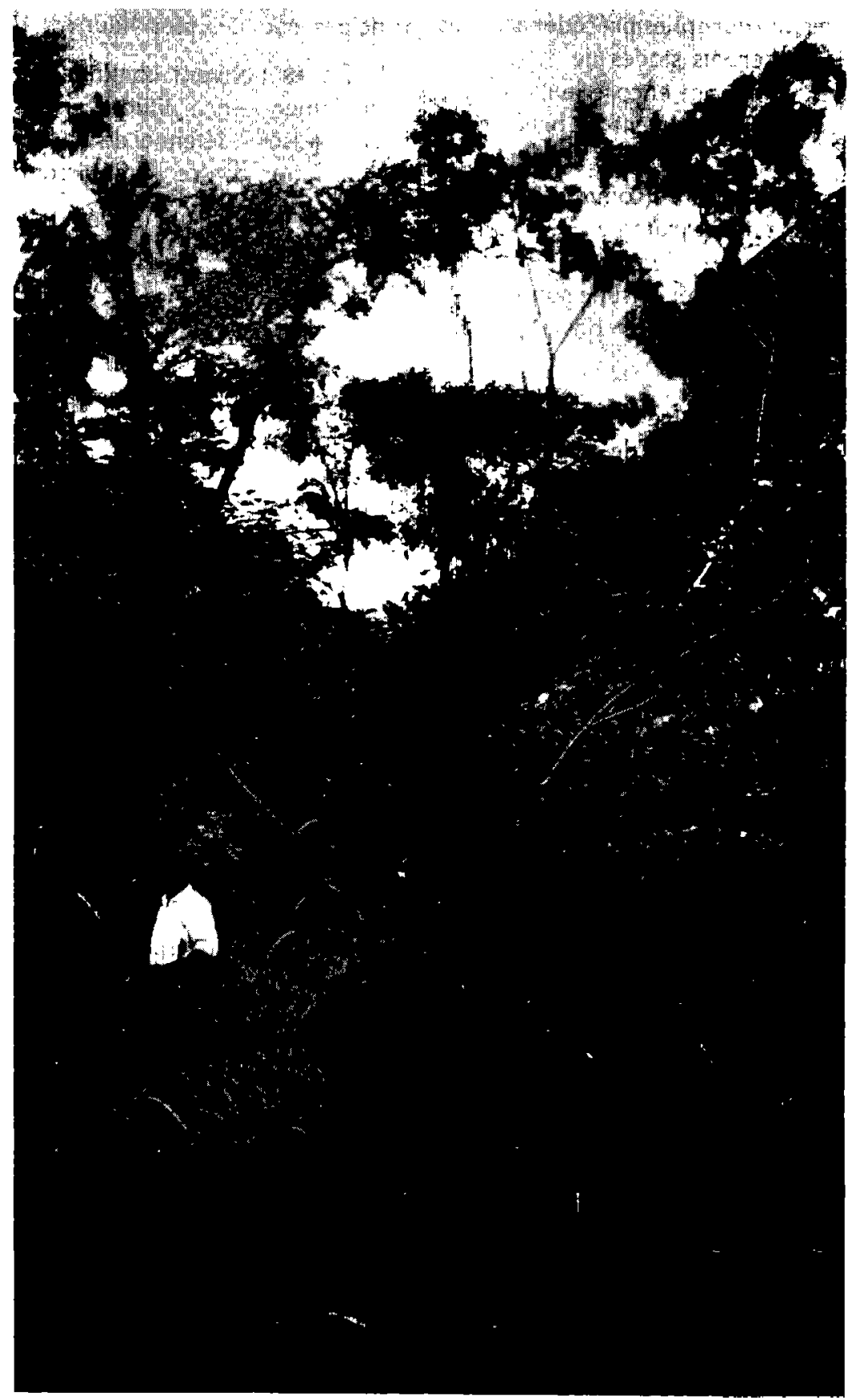

Environ $1 \mathrm{~km}$ plus loin. sur la brefelle, à gauche de la piste. Harungana nombreux, mais à part élancés au-dessus d'un tapis assez dense de hautes graminées (Panicum Arundinella). Même type d'exploitation que sur lo vue précédente. 


\section{PROGRAMME D'ACTION CONCERNANT L'ÉTUDE ET LA CONSERVATION DES PATURAGES}

On a vu dans les paragraphes précédents que, si l'on connaît les différents stades de dégradation des pâturages, on ignore encore leur extension en superficie ef bien souvent le processus précis d'évolution de la végétation.

Nous avons donc élaboré, en commun avec le service de l'élevage, un programme qui serait à mettre en cuvre en premier lieu dans le secteur occidental. II faudra envisager ensuite son application à l'ensemble des régions pastorales. II comporte les points suivants:

A) Reconnaissance ef inventaire précis des pâturages et des différents stades de dégradation Il est indispensable de connaître quelles sont actuellement les ressources pastorales et de dresser, à cet effet, un inventaire cartographique, à l'échelle du : 50.000e par exemple. Ce travail sera grandement facilité par l'existence d'une couverture photographique aérienne au 1 : 25.000e qui doit être exécutée sous peu dans le secteur occidental.

On pourra ainsi reconnaître :

A. Les zones de savane intacte, avec les différents types de végétation.

$B$. Les différents types de végétation sousexploités ou pâturés irrégulièrement et peu modifiés par l'action du bétail.

C. Les zones régulièrement pâturées, avec tapis herbacé amélioré, en notant sa composition botanique ou du moins les espèces dominantes.

D. Les zones dégradées, en distinguant différents types en fonction de la nature de la végétation secondaire, de l'étendue des dénudations et des phénomènes d'érosion.

Des notations secondaires donneront des indications sur la nature et la densité de la strate ligneuse et sur le degré d'embuissonnement, par des essences savanicoles ou forestières.

Une reconnaissance pédologique devra être menée parallèlement qui pourra préciser la nature des sols, et leur état d'évolution, en particulier celui de la couche superficielle humifère.
Cette carte permettra ainsi de délimiter en toute connaissance de cause les zones d'intérêts divers et de définir un programme d'exploitation de la zone cortographiée en fonction des principes énoncés plus haut :

A. Zones pouvant continuer à être pâturées normalement, en instituant un système de rotations de mise en défens et de brôlages périodiques, afin de lutter contre l'embuissonnement.

B. Zones ayant subi un début de dégradation ; à faire pâturer avec des charges faibles ou à mettre en défens pour de courtes périodes.

C. Zones très dégradées, à mettre en défens pour des périodes plus ou moins longues et sur lesquelles des interventions seraient à envisager.

Sur les zones exploitables, on organisera un système de rotation sur des pâturages délimités dans la mesure du possible par des frontières naturelles, rivières, galeries forestières, etc...

Le service de l'élevage, par une action soutenue pendant de nombreuses années, a su gagner la confiance totale des éleveurs. II lui sera sans doute possible d'attribuer à chacun une zone de parcours et de faire respecter cette attribution. Un tel système permettrait aussi dans de nombreux cas, de prolonger la durée d'exploitation des pâturages de saison sèche, en évitant le retour précipité des éleveurs désirant s'installer sur les meilleurs pâturages de saison de pluie.

Une telle organisation demandera évidemment un surcroît de personnel de surveillance. Le service pense créer à cette fin des agents africains qui seront pour les pâturages l'équivalent des infirmiers pour les questions sanitaires.

B) Expérimentation concernant l'évolution de la végétation et les modes possibles d'intervention

10 Evolution de la végétation et capacité de charge des pôturages.

En ce qui concerne l'évaluation de la capacité de charge, les méthodes seront décrites à propos de l'étude du programme des stations d'élevage. Il convient cependant de faire remarquer dès maintenant que l'application de ces méthodes aux pâturages extensifs est' délicate : souvent, la végétation n'est pas homogène, ce qui impose un échantillonnage considérable ; il faudrait aussi tenir comple des plantes normalement refusées. 
L'étude de l'évolution repose soit sur un échantillonnage périodique de la végétation, soit sur l'observation et le complage périodique des plantes situées sur des zones délimitées de façon permanente. De telles zones devront être placées dans tous les types de végétation dont on voudrait faire l'étude détaillée. Le nombre de points d'observation sera fonction de l'hétérogénéité des pâturages.

a) Echantillonnage. Cetfe méthode consiste à prélever la végétation par coupe sur de petites superficies (de l'ordre de $1 \mathrm{~m}^{2}$ ) et à en déterminer la composition par comptage ou pesée des différentes espèces. On peut utiliser par exemple un cadre en bois d'un mètre de côté que l'on pose sur le terrain un certain nombre de fois dans la zone à étudier. On prélève ce qui est à l'intérieur. La précision sera d'autant plus grande que le nombre de prélèvements sera plus important. II est possible de faire, soit un échantillonnage statistique sur l'ensemble d'une zone assez vaste, soit de choisir et de repérer des plages homogènes pour en suivre l'évolution.

b) Zones d'observation permanente. Elles permettent de suivre de façon précise l'évolution de la végétation et de déceler tous les changements intervenant dans la flore. On procède par repérage et comptage périodique des plantes sur les mêmes points.

On peut utiliser un carré de 2 mètres de côté, matérialisé sur le terrain par 4 bornes enfoncées suffisamment dans le sol pour ne pas gêner le bétail et on repère sur un dessin la position des plantes, leur superficie, celle des espaces dénudés.

On peut aussi repérer sur le terrain une ligne, de 20 mètres de long par exemple, en tendant un double décamètre métallique entre deux piquets fixes et on note la position de toutes les plantes situées dans un couloir large de 5 centimètres, pris à la verticale de la ligne.

kci encore, la précision des résultats sera fonction du nombre de carrés ou de lignes d'observation.

La mise en œuvre de ces méthodes demande évidemment du temps et exige un personnel connaissant parfaitement la flore de la région. II est cependant possible de simplifier le travail en classant les plantes en un certain nombre de groupes ayant chacun une signification déter- minée en ce qui concerne les problèmes fourragers :

1. Graminées pérennes de la savane naturelle ayant une bonne valeur fourragère (Hyparrhenia, Andropogon, Beckeropsis, Setaria).

II. Graminées pérennes de valeur fourragère médiocre :

a) Plantes de la savane naturelle (Loudetia spp. Urelytrum thryrsoides, Tristachya sp.).

b) Plantes secondaires (Imperata cylindra, Panicum phragmitoides, Urelytrum incomplctum).

III. Graminées annuelles:

a) Appétées (Digitaria spp. Eleusine indica...).

b) Non appétées (Sporobolus spp.).

IV. Plantes non graminéennes herbacées ou sub-ligneuses.

a) Espèces de la savane (Indigofera, Eriosema spp. Hypoestes cancellata).

b) Espèces secondaires (Amaranthus spinosus, Achyranthes aspera, etc...).

V. Rejets ligneux, arbustes, semis forestiers.

a) Espèces de savane (Annona arenaria, Burkea africana, Adenodolichos paniculatus).

b) Espèces forestières (Harungana madagascariensis, Croton macrostachyus).

La première méthode d'échantillonnage par coupe est la plus facile à utiliser. Le comptage ou le repérage par dessin est plus délicat, surtout avec la végétation cespiteuse des savanes ou celle plus étalée, des pâturages : il faudra distinguer dans ce dernier cas pour chaque plante la surface réellement enracinée et celle qui est recouverte par des stolons, des feuilles ou des tiges rampantes.

$2^{0}$ Possibilités d'intervention pour lo lutte contre l'invasion des mauvaises herbes.

On a vu que la mise en défens de pâturages dégradés risquait de favoriser la 'prolifération d'espèces peu intéressantes, telles que Panicum phragmitoides, ou de mauvaises herbes telles que Solanum torvum.

Il serait donc intéressant d'être à même de contrôler et de diriger l'évolution de la flore sur. les pâturages. Les essais pourraient être menés sur des petites parcelles protégées du bétail, de l'ordre de quelques ares de superficie: 
- influence de la simple mise en défens sur différents types de végétation ;

- influence du brôlage sur différentes plantes ou divers types de végétation ;

- essais d'ensemencement dans différents milieux et avec diverses espèces. On peut utiliser des plantes annuelles, propres à s'installer dans des terrains dégradés. Elles ne sont pas toujours intéressantes par elles-mêmes, mais permettront l'implantation ultérieure de plantes meilleures dans un milieu qu'elles auront amélioré.

Dans des pâturages relativement peu dégradés on pourra essayer d'implanter directement des espèces pérennes.

- essais de lutte anti-érosive par des travaux légers destinés à favoriser les dépôts colluviaux, avec ou sans semis de plantes fourragères.

Les différentes plantes à essayer pourront être testées au préalable en station.

L'observation de la végétation le long d'une ligne matérialisée paraît être la méthode la mieux appropriée pour suivre de tels essais.

3o Essais de lutte contre l'embuissonnement.

Le feu est évidemment le moyen le plus pratique et le plus économique, mais on a vu qu'il n'est utilisable que si le tapis herbacé est assez dense et bien fourni en espèces pérennes de grande taille.

Irès souvent, il pourra être intéressant de prévoir un premier temps de mise en défens pour permettre d'abord la reconstitution du tapis herbacé. Les moyens d'éradication manuels, mécaniques ou chimiques pouvant être employés sont les suivants :

- abattage à la main.

- abattage au tracteur, à la chaine (deux engins tirent entre eux une lourde chaîne qui couche les arbres) ou par poussée.

A noter à ce propos que l'Harungana madagascariensis a un enracinement superficiel et peut être facilement couché : lorsque ces arbres sont isolés en savane, ils sont fréquemment déracinés par le vent,

- Iutte chimique par hormones désherbantes.

Ce procédé a été utilisé, au Gabon par exemple, pour détruire les gros arbres de la forêt en vue de plantations d'Okoumé.

On pulvérise le produit soit directement sur l'écorce, soit sur une couronne écorcée, soit sur des entailles pratiquées à la hache sur un ou deux cercles superposés tout autour du tronc (entailles malaises).

On a utilisé un certain nombre de produits, à base de 24D ou de 245T : Phytosyl-Bois (Protex, Anvers)-Phortox (Fison-chemicals)-Rhodia débroussaillant ef Rhodia 600 (Rh.-Poulenc)-P 80 (Procida)-Débroussaillant Péchiney-Progyl.

Les résultats obtenus sont très inégaux selon les essences et les techniques utilisées. Dans le cas présent, toute l'expérimentation serait à reprendre à la base (1).

Si l'on procède par abattage, il faut s'attendre à une importante prolifération de rejets de souche: il faut donc pouvoir brûler ou entretenir la coupe par la suite.

Dans les conditions de l'élevage extensif, on ne peut pas se permettre de faire de gros investissements sur les pâturages. Qu'il s'agisse de l'amélioration du tapis herbacé ou de lutte contre l'embuissonnement, la question du prix de revient et de la rentabilité ne devra jamais être perdue de vue.

Il est probable que dans de nombreux cas il faudra se contenter d'utiliser les armes naturelles: mise en défens et brûlage.

Par contre, pour l'exécution de la première partie du programme, reconnaissance et exploitation rationnellc des pâturages, la misc en œuvre de moyens importants est justifiée : c'est l'avenir de l'élevage en République Centrafricaine qui est en jeu. Le troupeau représente un capital ef un revenu tel qu'il serait coupable de le négliger.

\section{EXPÉRIMENTATION SUR LES STATIONS D'ÉLEVAGE}

Grâce au personnel et au matériel dont on dispose sur ces stations, il sera possible de mener à bien toute expérimentation détaillée concernant l'exploitation des pâturages naturels.

De plus, on dispose maintenant, dans les zones guinéennes et soudano-guinéennes du moins, de plantes fourragères de valeur et de techniques permettant, soit d'améliorer considérablement

(1) Voir à ce sujet : Catinat ef Leroy-Deval. - Essais de destruction de la forêt dense par empoisonnement au Gabon. Bois et Forêts des Tropiques, janv.-fév. 1960, pp. 3-16. 
un pâturage naturel, soit. même de créer des pâturages artificiels de haute qualité.

La mise au point locale de ces techniques revêt pour l'agriculture un intérêt exceptionnel. Une exploitation rationnelle, préoccupée avant tout du maintien et de l'amélioration de la fertilité des terres, ne se conçoit en effet qu'avec l'association de l'élevage à la culture.

Le problème est actuellement résolu dans l'agriculture africaine par un nomadisme perpétuel, du fait du recours à la jachère naturelle de très longue durée.

Au moment où l'on introduit dans les villages un élevage bovin sédentaire, son association à la culture, grâce à des rotations utilisant des jachères pâturées ou des cultures fourragères, serait à envisager.

\section{A) Ełude des pâturages et appréciation de leur productivité}

Les méthodes destinées à suivre l'évolution de la végétation décrites plus haut sont applicables en station. Elies pourront l'être même avec beaucoup plus de précision et de facilité. II en est de même en ce qui concerne l'étude de la productivité des pâturages.

Deux méthodes sont utilisables : la première consiste à installer un troupeau sur un pâturage, en adaptant constamment la charge à la superficie de façon à ce que toute la pousse soit broutée.

On note le nombre de journées de pâturages effectuées, ef le gain de poids des animaux. Un tel système nécessite un cloisonnement du pâturage de façon à pouvoir l'exploiter en rotation.

La deuxième méthode consiste à couper périodiquement l'herbe sur une surface déterminée, à la peser et à en déterminer la valeur fourragère.

La mise en œuvre de la première méthode n'appelle pas de remarque particulière. Elle nécessite des moyens de clôture et un troupeau assez maniable. On choisira dans la mesure du possible un terrain ayant une végétation aussi homogène que possible.

En ce qui concerne la deuxième méthode, la seule pesée périodique du fourrage produit donne déjà d'appréciables renseignements ef permet d'établir avec plus de précision un programme de rotation.

Nous avons indiqué déjà les précautions à prendre pour le prélèvement d'échantillons des- tinés à l'analyse. Chaque prélèvement ne donnera de renseignements que sur la valeur du pâturage au moment où il a été fait. On pourra effectuer, soit un prélèvement moyen sur l'ensemble de la coupe, soit un prélèvement par espèce, en notant les proportions relatives de chacune.

La productivité de l'herbe peut dépendre dans une large mesure de la fréquence des coupes. II sera done nécessaire d'expérimenter différents rythmes d'exploitation.

Enfin, il faudra tenir compte dans les calculs de la présence éventuelle de plantes qui seraient normalement refusées par le bétail.

Cette expérimentation peut être menée à l'aide d'enclos ou de cages grillagées faciles d̀ déplacer et couvrant des surfaces de l'ordre de $4 \mathrm{~m}^{2}$. Installées sur un pâturage, on les déplace périodiquement sur un nouvel emplacement fraîchement pâturé ou coupé et on récolte le fourrage produit sur l'emplacement précédent.

\section{B) Conduite rationnelle du pâturage}

Le but recherché est une utilisation régulière des herbages en toute saison, sans' surcharge ni sous-exploitation locale.

Le moyen le plus rationnel d'y parvenir est une exploitation par rotation, la superficie totale du pâturage étant divisée en un certaịn nombre de parcs utilisés chacun à tour de rôle pendant que I'herbe repousse dans les autres. Pour obtenir une pâture régulière il est plus avantageux d'utiliser sur une surface donnée des charges fortes pendant peu de temps que des charges faibles pendant une longue période.

Dans les pays à saison sèche marquée, comme c'est le cas ici, la végétation est très ralentie en dehors des pluies et la superficie nécessaire est alors beaucoup plus considérable. On résout le problème par des brûlages échelonnés, avant la période sèche, et pendant toute la durée de celle$\mathrm{ci}$, afin d'avoir constamment une repousse jeune à sa disposition.

Un système de rotation rigoureux implique nécessairement un cloisonnement des pâturages : en pratique ceci ne sera possible que dans la mesure où le prix de la clôture esti payé par le surcroît de charge ou de productivité apporté par l'exploitation rationnelle du pâturage et par son amélioration. 
Au delà de cette limite, c'est au berger qu'il faudra demander de diriger la rotation en conduisant successivement le troupeau dans les différentes parties du pâturage. Sa tâche sera facilitée par des distributions de sel faites dans des abris mobiles que l'on déplacera au fur et à mesure des besoins.

\section{C) Entretien et amélioration des pâturages}

Une exploitation bien conduite améliore la structure du pâturage et, la conserve en bon état par le simple jeu d'une rotation judicieuse ef d'une charge de bétail correcte. Un certain nombre d'interventions sont cependant susceptibles d'augmenter encore ces bonifications.

10 Déboisement des pâturages.

La première conséquence de cette opération se traduit par une augmentation de la surface utilisable. Elle permettra de plus une meilleure utilisation d'engins mécaniques.

A Bovar, on a entrepris le dessouchage de certains parcs. C'est une opération longue et onéreuse. De plus, sur la terre remuée par l'opération, se développent de nombreuses mauvaises herbes qui auront tendance à s'installer partout dans les espaces laissés libres entre les touffes d'herbe.

Une meilleure technique consisterait sans doute à couper arbres et arbustes au ras du sol ; la végétation graminéenne reste intacte et le passage d'engins mécaniques du type Rotarycutter n'est pas entravé. Celui-ci de plus détruira facilement les rejets que l'on pourrait aussi tenter d'éliminer par un traitement des souches aux hormones. Elles seront peu à peu décomposẻes et attaquées par les termites.

\section{Utilisation d'engins mécaniques.}

Le plus intéressant est le rotary-cutter, constitué par une lame coupante tournant horizontalement et portée par un bâti monté sur rove. L'appareil peut être tiré par un tracteur de moyenne puissance muni d'une prise de force. II joue le rôle d'une faucheuse, mais plus robuste, coupe facilement les rejets ligneux, hache ef répartit l'herbe coupée sur tout le terrain. L'action du rotary-cutter est analogue à celle d'une pâture régulière et provoque une augmentation considérable de la densité du tapis herbacé, tout en éliminant les refus. C'est l'instrument idéal pour préparer et entretenir un pâturage.

Selon une suggestion de $M$. Blanchon (technique employée à la station de Sotuba au Soudan), le rotary peut êtrc utilisé pour ouvrir et entretenir dans les parcs un certain nombre de bandes, de 30 à 50 mètres de large, alternant avec des bandes laissées intactes. Les bandes nettoyées seront utilisées en saison des pluies et exploitées en rotation. Leur nombre sera fónction de la charge et les zones restantes servent de réserve: on peut y ouvrir de nouvelles bandes en cas de besoin. On les brûlera en saison sèche pour augmenter la superficie du pâturage.

Le brush-cutter est un rouleau à lames tranchantes longitudinales, du type débroussailleuse landaise. II peut être utilisé pour ouvrir des pâturages dans une végétation très haute et encombrée de buissons : il écrase et tronçonne les tiges, favorisant le thallage et provoquant le bouturage de certaines espèces. Cet appareil serait certainement intéressant pour préparer des pâturages à partir de peuplements d'herbe à éléphants (Pennisetum purpureum). En brisant lcs tiges, en les enterrant partiellement pour les bouturer, il provoquerait certainement une augmentation considérable de la densité réelle de la végétation au niveau du sol.

Chaque station peut en outre avoir ses problèmes particuliers à étudier.

Un des plus importants est celui du Panicum phragmitoides, herbe fort mal appétée qui envahit les anciennes jachères et les pâturages surexploités. C'est le cas en particulier à Bossembélé sur les zones situées au nord de la route de Bangui : on a là de vastes peuplements de Panicum, en mélange avec Brachiaria brizantha ef Hyparrhenia confinis: ces deux espèces, très appétées constituent pratiquement l'essențiel du pâturage sous forme de taches plus ou moins étendues en peuplement dense.

II serait intéressant de rechercher une technique propre à assurer le remplacement du Ponicum par ces espèces.

\section{D) Enrichissement de la flore des pâturages et cultures fourragères}

En vue d'augmenter la valeur des pâturages, on "peut envisager d'y introduire une certaine 
quantité de plantes fourragères. Il s'agit donc de semer ou de planter ces nouvelles espèces, mais en touchant le moins possible à la végétation d'origine.

Les graminées de la savane sont, en effet, très sensibles d̀ toute façon culturale et se trouvent facilement remplacées par des mauvaises herbes. On pourra utiliser un instrument à disque, genre déchaumeuse lourde, mais en réglant l'ouverture des disques pour que le sol soit légèrement ouvert, sans que les mottes soient retournées. La plante fourragère est simplement semée à la volée.

Une des plantes les plus intéressantes à introduire dans ces conditions est le STYLOSANTHES GRACILIS, légumineuse originaire d'Amérique du Sud, dont les qualités sont très voisines de celles d'une luzerne: appétabilité, rendèment, comportement au paturage, résistance d la saison sèche, valeur fourragère sont autant de facteurs intéressants. Le Stylosanthes fleurit ef fructifie en saison sèche ; les graines peuvent être récoltées à la main ou à la moissonneuse-batteuse. Leur pouvoir germinatif est généralement faible du fait de l'imperméabilité des téguments. On peut l'augmenter considérablement par un traitement chimique à l'acide sulfurique, ou mieux par une usure mécanique : un polisseur à Paddy à lames de cuir utilisé à cet effet d̀ la station de I'IRCT de Madingou (République du Congo) donne entière satisfaction.

Pour les enrichissements de pâturages, le Stylosanthes se sème à raison de 3 à 5 kilos à I'hectare.

Il est ensuite nécessaire d'entretenir le pâturage au Rotary-cutter, au moins pendant les premiers mois, afin d'éviter une trop forte concurrence des graminées. Le feu doit être évité, le Stylosanthes $y$ étant très sensible. Le pâturage peut être mis en exploitation environ un an après le semis.

D'après les expériences faites au Congo belge et dans la vallée du Niari, le Stylosanthes s'installe et se développe bien dans ces conditions, arrivant même à supplanter partiellement les graminées.

D'autres plantes pourraient sans doute être utilisées de la même façon. Citons en particulier deux graminées, MELINIS MINUTIFLORA et BRACHIARIA BRIZANTHA, toutes deux bien appétées, résistant bien à la saison sèche et se comportant correctement au pâturage. Des essais pourraient également être tentés avec des Hyparrhenia $(H$. diplandra ou $H$. rufa) pour améliorer les peuplements à base de Panicum phragmitoides.

Ces mêmes plantes, le Stylosanthes en particulier, peuvent être utilisées en cultures pures. On obtient ainsi des stocks fourragers de grande valeur pouvant servir d'appoint pour la saison sèche, ou en cas de besoin momentané. De tels herbages peuvent également servir avantageusement à «finir » des animaux destinés à la vente.

Certaines plantes, le Stylosanthes entre autres, sont aussi bien utilisables en vert qu'à l'état sec. Enfin on peut même envisager la fabrication d'ensilages. On les réussit parfaitement en fosse, avec des graminées, qu'il faut cependant avoir soin de tasser fortement. On peut utiliser du maïs vert, de l'herbe à éléphant, ou de l'herbe jeune de savane.

Il est essentiel de semer la plante fourragère sur un terrain bien préparé et propre afin d'éviter une trop grande concurrence des mauvaises herbes.

On pourra exécuter un labour, deux passages croisés de cultivateur, puis hersage et éventuellement passage d'un rouleau. La plante fourragère succédera avantageusement à une autre culture qui aura déjà permis un nettoyage du sol. La plante fourragère elle-même pourra être semée en interligne d'une plante abri à court cycle, maïs ou paddy par exemple, qui assurera lá couverture du sol dans les premiers temps. Enfin, elle sera semée en ligne, ce qui permet un entretien plus facile de la culture. En général, la prairie artificielle ne sera pas utilisable comme pâturage avant la deuxième année.

Différentes plantes pourront être essayées, choisies dans la flore locale ou importées. II sera nécessaire, au préalable, de tester leur valeur sur les points suivants :

- Possibilités de multiplication (production de graines ou de boutures ef faculté germinative ou de reprise).

- Rendement.

- Résistance à la sécheresse.

- Comportement au pâturage.

- Valeur nutritive, appétabilité.'

Les mélanges d'espèces, particulièrement de légumineuses et de graminées sont, à priori, plus intéressants que les cultures pures. 
Parmi les légumineuses, on peut essayer Stylosanthes gracilis et Centrosema pubescens. La première de ces deux espèces est certainement la plus intéressante, du moins en climat assez humide sinon elle a tendance à devenir trop ligneuse. Elle se multiplie bien par semis, plus difficilement par bouturage de tiges vertes.

II serait bon également d'explorer les possibilités de la flore locale.

Parmi les graminées, le choix de plantes à essayer est plus grand. On peut distinguer:

- Des plantes annuelles susceptibles de donner rapidement d'assez grandes quantités de fourrage à récolter (foin, ensilage) : Ericholaena rosea, Pennisetumi subangustum, Rottboellia exaltata.

- Des plantes pérennes à pâturer ou pour récolte de fourrage :

- par multiplication végétative: Panicum maximum (éclats de souches; les graines sont généralement parasitées et rendent le semis impossible), Pennisetum purpureum (boutures de tiges). Brachiaria brizantha (éclats de souches: la récolte de graines est cependant possible, mais généralement avec un rendement très faible).

- par semis : Beckeropsis uniseta, Andropogon tectorum et A. gayanus, Hyparrhenia diplandra. Melinis minutiflora.

Cette dernière espèce est particulièrement intéressante. Elle semble donner d'excellentes associations avec le Stylosanthes. Avec Brachiaria brizantha, ce sont les deux espèces qu'il serait probablement le plus intéressant d'essayer.

Le Bourgou enfin (Echinochloo stagnina) pour- rait sans doute être introduit dans les zones inondables à sol pas trop acide ni tourbeux. Les différents modes de multiplication restent encore à essayer.

\section{CONCLUSIONS}

Nous avons montré dans cette étude quels problèmes se posaient à propos de l'exploitation des pâturages en République Centrafricaine. Ils sont nombreux: reconnaissaance des zones utilisables, lutte contre les phénomènes de dégradation, amélioration des herbages naturels, cultures fourragères.

Nous espérons également avoir suffisamment mis l'accent sur l'urgence qu'il y avait à résoudre certains d'entre eux qui conditionnent pour une large part l'existence de l'élevage dans ce pays.

Tant dans l'intérêt des populations, que pour l'avenir économique de la R. C. A., cet élevage prend de jour en jour une importance plus capitale.

Le service de l'élevage, qui s'est préoccupé jusqu'ici essentiellemet, et à juste titre, des problèmes sanitaires; envisage maintenant de consacrer une part beaucoup plus grande de son activité à ces problèmes pastoraux et fourragers.

Il esl donc essentiel que soient mis à sa disposition les moyens qui lui permettront d'entreprendre cette action en se basant sur le programme qui a été exposé ici.

Office de la recherche scientifique et technique outre-mer.

\section{SUMMARY}

\section{Studies on Pasture and Animal Foodstuffs in the Central Africain Republic}

The author points out the problems pertaining to animal nutrition, to be solved in the Central African Republic, they are many ; survey of the pasture areas, soil conservation, pasture improvement and the fodder crops.

He stresses the urgent need for solving some of these problems which are fundamental to continued existence of an animal industry in this country.

Animal production becomes daily of more and more capital importance to the economic future of the Republic and in the interest of its peoples.

The Department of Animal Production up to the present, and rightly so, concerried with problems of animal health, should now allocate more time and attention to grazing and animal feeding problems. 


\section{RESUMEN}

\section{Estudio sobre pastos y cuestiones forrajeras en la republica centroafricana}

Nosotros presentämos en este estudio algunos problemas que aparecen en La Republica Centroafricana a proposito de la explotacion de pastos. Ellos son numerosos : reconocimiento de zonas utilizables, lucha contra la erosion, mejoracion de las especies espontaneas y cutlivos forrajeros.

Nosotros hemos remarcado ante todo nuestra atencion, sobre aquellos mas estrechamente relacionados con la existencia de explotaciones ganaderas en este pais.

Tanto por el interes general, como por el porvenir economico de la R. C. A. ; estas explotaciones adquieren cada dia mas importancia.

Los servicios agropecuarios que se han preocupado hasta aqui y acertadamente, de los problemas sanitarios, deberan desde ahora consagrar una parte mas importante de sus actividades a las cuestiones de pastos y forrajes. 